\title{
On stochastic stability of regional ocean models to finite-amplitude perturbations of initial conditions
}

\author{
Leonid M. Ivanov ${ }^{\mathrm{a}, \mathrm{b}, *}$, Peter C. Chu ${ }^{\mathrm{a}}$ \\ a Department of Oceanography, Naval Postgraduate School, Monterey, CA 93943, USA \\ b Department of Mathematics, University of Southern California, Los Angeles, CA 90089, USA
}

Received 13 September 2006; accepted 14 March 2007

Available online 20 March 2007

\begin{abstract}
We consider error propagation near an unstable equilibrium state (classified as an unstable focus) for spatially uncorrelated and correlated finite-amplitude initial perturbations using short- (up to several weeks) and intermediate (up to 2 months) range forecast ensembles produced by a barotropic regional ocean model. An ensemble of initial perturbations is generated by the Latin Hypercube design strategy, and its optimal size is estimated through the Kullback-Liebler distance (the relative entropy). Although the ocean model is simple, the prediction error (PE) demonstrates non-trivial behavior similar to that existing in 3D ocean circulation models. In particular, in the limit of zero horizontal viscosity, the PE at first decays with time for all scales due to dissipation caused by non-linear bottom friction, and then grows faster than (quasi)exponentially. Statistics of a prediction time scale (the irreversible predictability time (IPT)) quickly depart from Gaussian (the linear predictability regime) and becomes Weibullian (the non-linear predictability regime) as amplitude of initial perturbations grows. A transition from linear to non-linear predictability is clearly detected by the specific behavior of IPT variance. A new analytical formula for the model predictability horizon is introduced and applied to estimate the limit of predictability for the ocean model.
\end{abstract}

(C) 2007 Elsevier B.V. All rights reserved.

Keywords: Oceanography; Wind-driven circulation; Current prediction; Stochastic stability; Statistical analysis

\section{Introduction}

A number of recent theoretical studies have demonstrated that the robust dynamical regimes (attractors) of oceanic circulation often present a combination of (quasi-stable) equilibrium

\footnotetext{
* Corresponding author at: Department of Oceanography, Naval Postgraduate School, Monterey, CA 93943, USA. Tel.: +1 831656 3257; fax: +1 8316563686 .

E-mail address: 1mivanov@nps.edu (L.M. Ivanov).
} 
states and transient dynamics between these flow configurations (Berloff and McWilliams, 1999; Schmeits and Dijkstra, 2000; Stanev and Staneva, 2000; Sura et al., 2001; Lermusiaux et al., 2006 among others). For example, Eremeev et al. (1992) and Stanev and Staneva (2000) identified single, double, and multiple basin-scale current gyres observed in the Black Sea as quasi-stable equilibrium states, for which transient dynamics were induced by baroclinic instability and mesoscale anti-cyclonic eddy activity. Another example that was well documented in observations and numerical models is the path variation of the Kuroshio south of Japan (Masuda et al., 1999).

Clearly, an equilibrium state influences the phase-spatial organization of the local prediction error growth rate, i.e., it organizes the local predictability for a forecast model. Therefore, the knowledge on how small- and finite-amplitude perturbations evolve near this equilibrium state is important for understanding regional model predictability and identifying the persistence of circulation ocean and atmospheric patterns with oscillations near equilibrium states (Robinson et al., 1996).

The primary goals of the proposed study are (1) to understand what mechanism(s) can form the statistics of finite-amplitude prediction error (PE) near an unstable equilibrium state identified as an unstable focus, (2) to check how quickly such statistics depart from Gaussian (if such a departure exists) for the short- (up to several weeks) and intermediate (up to a couple of months) range forecasts, and (3) how to quantify PE statistics for perfect models with initial conditions corrupted by finite-amplitude stochastic perturbations.

The computations presented below assume a perfect model scenario with stochastic perturbations in initial conditions. A non-linear barotropic model of wind-driven circulation in an idealized basin is used to understand evolution of prediction error. Although this model seems to be too simple in comparison with large state of the art oceanic models, it describes a generic system with many degrees of freedom while not requiring large computer resources. The latter feature allows us to generate non-rank deficient forecast ensembles and strongly reduce errors in determination of appropriate distributions in comparison with small ensemble integration.

Predictability in real-time systems can be qualified in many ways (for example, see Smith et al., 1999). The proposed study defines model predictability in the stochastic stability context. The stochastic stability addresses effects of random perturbations on trajectories of a dynamical system and estimates its stability in terms of probabilistic measures, such as expected values or distribution functions (Freidlin and Wentzell, 1998). In general, the stochastic stability and predictability differ from one another. However, if a time scale quantifies the model predictability, and if this scale indicates the time when the forecast uncertainty exceeds some boundary or when information on the initial condition is lost, the stochastic stability and predictability are interchangeable. Since these time scales are widely used in meteorology (see, for example, Toth, 1991) and oceanography (Robinson et al., 1996), the stochastic stability concept seems to be a useful tool for the predictability analysis of large hydrodynamic models.

The loss of superposition and the extreme inhomogeneity common in non-linear hydrodynamic models require applying local measures of predictability and corresponding time scales (see Lorenz, 1965; Benzi and Carnevale, 1989; Ivanov et al., 1994; Boffetta et al., 1998; Smith et al., 1999; Mu et al., 2004, and references thereof). It is widely held that time scales are related to the inverse of the largest Lyapunov exponent estimated by the tangent linear models in assumption of small-amplitude initial perturbations. The linear approach gives reasonable estimations of model predictability in many practical cases. However, it cannot provide critical boundaries on finite-amplitude stability of the thermohaline ocean circulation. 
In regional ocean modeling, neither initial perturbations nor prediction errors are small. Therefore, the linear predictability regime, where the PE grows (quasi)-exponentially or even faster than exponentially, may be quickly replaced by the non-linear predictability regime, for which the predictability time is much larger than the inverse of the leading Lyapunov exponent (Aurell et al., 1996; Lorenz, 2005 and others).

To quantify both the linear and non-linear predictability the proposed study uses the so-called irreversible predictability time (IPT) originally introduced by Ivanov et al. (1994). Chu et al. (2002) demonstrated the capability of IPT for the analytical estimate. They have obtained an analytical formula for the mean IPT and variance of IPT for Lorenz-84 atmosphere model (Lorenz, 1984). Here, we would like to show how IPT statistics change with a transition from linear to nonlinear predictability regimes, and to find what kind of PE statistics may accompany the non-linear predictability regime.

The paper is organized as follows. Section 2 briefly discusses IPT and its statistics. Section 3 analyzes the specificities of the reference solution reproduced by a barotropic regional ocean model. The ensemble of stochastic perturbations added to the initial conditions is described in Section 4. Section 5 depicts the optimal ensemble size of stochastic perturbations using the Kullback-Leibler distance. Section 6 discusses the initial PE decay due to viscosity damping. Section 7 studies the response of the model to finite-amplitude initial perturbations, and analyzes a basic feature of the PDF of IPT (denoted $\tau$-PDF), such as non-Gaussianity. Section 8 gives evidence for using a three-parameter Weibull distribution as IPT statistics in the non-linear predictability regime. Variations of mean IPT and its variance for transition from the linear to non-linear predictability regime are discussed in Section 9. Section 10 estimates the model predictability horizon (the maximum predictability time for the given statistics of initial perturbations). Section 11 summarizes the obtained results.

\section{Irreversible predictability time}

Let the prediction error $\mathbf{Z}(\mathbf{x}, t)$ be defined as a difference between the reference solution $\hat{\mathbf{Y}}(\mathbf{x}, t)$ (i.e., the solution of a perfect model without errors in initial conditions; Lacarra and Talagrand, 1988) and an individual forecast $\mathbf{Y}(\mathbf{x}, t)$ :

$$
\mathbf{Z}(\mathbf{x}, t)=\hat{\mathbf{Y}}(\mathbf{x}, t)-\mathbf{Y}(\mathbf{x}, t), \quad \mathbf{Z}_{0}=\mathbf{Z}\left(\mathbf{x}, t_{0}\right),
$$

where $(\mathbf{x}, t)$ are spatial coordinates and time and $\mathbf{Z}_{0}$ refers to the initial perturbations. $\mathbf{Y}(\mathbf{x}, t)$ is the state vector, which may include velocity, temperature, salinity and other fields.

The ocean-atmospheric model predictability is often quantified by the weighted relative root mean square error (see Robinson and Haidvogel, 1980; Holland and Malanotte-Rizzoli, 1989; Brasseur et al., 1996; Robinson et al., 1996; Wirth and Ghil, 2000 among others) written as

$$
J\left(\mathbf{Z}_{0}, \mathbf{W}, t\right)=(\mathbf{Z}, \mathbf{W Z}) \cdot J_{\text {norm }}^{-1}(t),
$$

where $\mathbf{W}$ is the weight matrix, $(\cdot, \cdot)$ is the inner product, and the function $J_{\text {norm }}(t)$ is specified from the physics.

The IPT is defined as the time $\tau$, at which $J\left(\mathbf{Z}_{0}, \mathbf{W}, t\right)$ reaches a predetermined level $\bar{\varepsilon}^{2}$ for the first time:

$$
\tau\left(\mathbf{Z}_{0}, \mathbf{W}, \bar{\varepsilon}\right)=\inf _{t \geq 0}\left(t \mid J\left(\mathbf{Z}_{0}, \mathbf{W}, t\right)>\bar{\varepsilon}^{2}\right),
$$

where $\bar{\varepsilon}$ is a non-dimensional tolerance level (accepted prediction accuracy). 

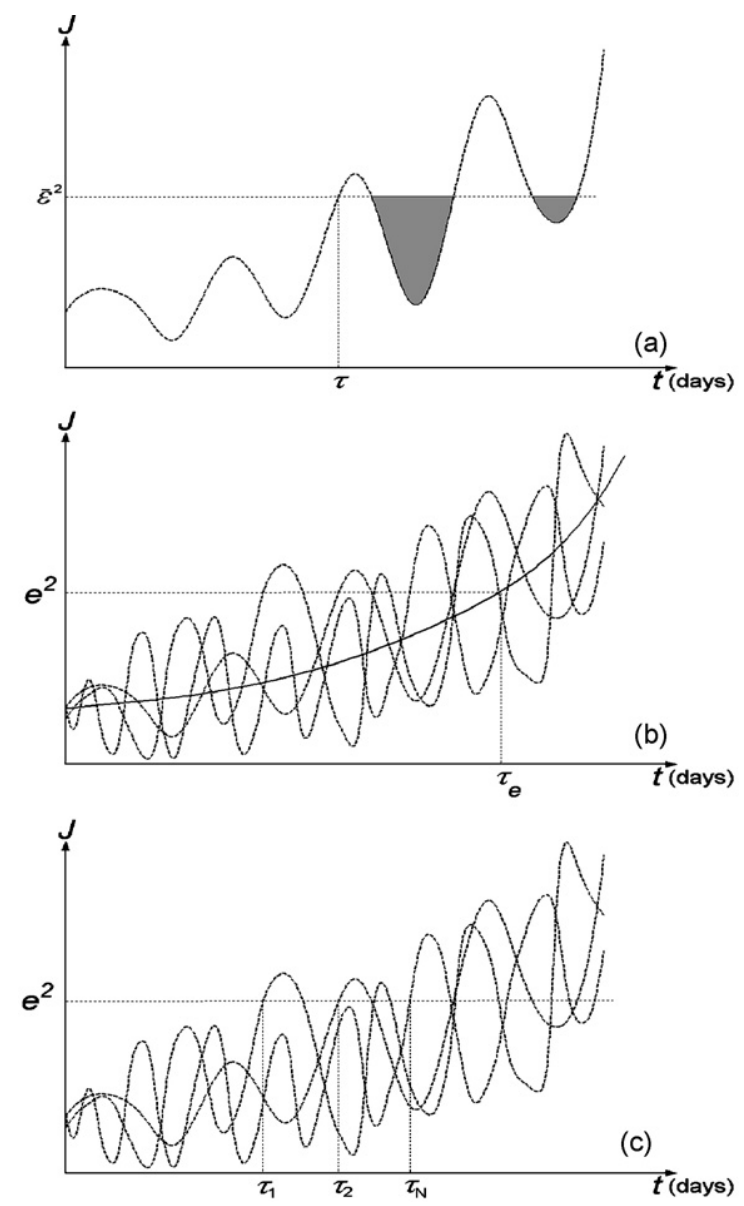

Fig. 1. The IPT and e-folding time for an oscillating prediction error. (a) IPT $(\tau)$ computed in an individual forecast. Shaded zones show returns of model predictability. (b) An ensemble of $J$ (dashed curves), the ensemble averaged $J$ (solid curve) and the e-folding time $\left(\tau_{\mathrm{e}}\right)$, and (c) the ensemble of $J$ (dashed curves) and an appropriate ensemble of IPT ( $\tau_{1}, \ldots$, $\left.\tau_{N}\right)$.

This definition is illustrated by Fig. 1a. Clearly, the IPT defines the model predictability on the condition that any returns of model predictability (the shaded zones in Fig. 1a) do not contribute to the prediction skill.

The mean IPT differs from the e-folding or the doubling time when $J$ oscillates or is random. To compare, for example, the e-folding and the irreversible predictability time, we should suppose in Eq. (2) $J_{\text {norm }}=\left(\mathbf{Z}_{0}, \mathbf{Z}_{0}\right)$ and $\hat{\varepsilon}^{2}=e^{2}$. The e-folding time is the time when $\langle J\rangle \operatorname{crosses} e^{2}$ (Fig. 1b):

$$
\tau_{\mathrm{e}}(\mathbf{W})=\max _{t \geq 0}\left(t \mid\left\langle J\left(\mathbf{Z}_{0}, \mathbf{W}, t\right)\right\rangle \leq e^{2}\right),
$$

where the brackets $\langle\cdot\rangle$ denote the average over the ensemble of initial perturbations $\mathbf{Z}_{0}$.

The mean IPT for the same $e$ is computed by

$$
\langle\tau(\mathbf{W}, e)\rangle=\left\langle\inf _{t \geq 0}\left(t \mid J\left(\mathbf{Z}_{0}, \mathbf{W}, t\right)>e^{2}\right)\right\rangle,
$$


where the averaging is over the ensemble of IPTs $\left(\tau_{1}, \ldots, \tau_{N}\right)$ induced by the ensemble of $\mathbf{Z}_{0}$ (Fig. 1c). Chu and Ivanov (2005) pointed out that the mean IPT is the lower bound of e-folding time:

$$
\tau_{\mathrm{e}}(\mathbf{W}) \geq\langle\tau(\mathbf{W}, e)\rangle
$$

In practical applications, the ensemble generated $\tau-\operatorname{PDF}(F(\tau, \mathbf{W}, \bar{\varepsilon})$, hereafter, this is a PDF which corresponds to the given ensemble of initial perturbations), the cumulative distribution function of $\tau(\tau-\mathrm{CDF})$ :

$$
P\left(\mathbf{W}, \bar{\varepsilon}, t-t_{0}\right)=\operatorname{Prob}\left(\tau \geq t-t_{0}\right),
$$

and $\tau$-moments calculated by

$$
\left\langle\tau_{k}(\mathbf{W}, \bar{\varepsilon})\right\rangle=k \int_{t_{0}}^{\infty}\left(t-t_{0}\right)^{k-1} P\left(\mathbf{W}, \bar{\varepsilon}, t-t_{0}\right) \mathrm{d} t, \quad k=1 \ldots K,
$$

may be used to quantify model predictability. The first four unbiased $\tau$-moments determine $\tau$-mean $(\langle\tau\rangle), \tau$-variance $\left(\left\langle\delta \tau^{2}\right\rangle\right), \tau$-skewness (SK) and $\tau$-kurtosis (KU).

For simplicity, the further analysis supposes (a) to replace the weight matrix $\mathbf{W}$ by the identity matrix I, (b) to describe flow dynamics in a quasi-geostrophic approximation using the geostrophic stream function $\Psi$ (Pedlosky, 1987), and (c) to take $J_{\text {norm }}=(\hat{\Psi}, \hat{\Psi})$.

\section{The reference solution}

A shallow water circulation computed in a flat bottom semi-enclosed basin and forced by wind and water flux across its open boundary, is taken as the reference solution. Our model leaves out the effects of topographic and baroclinic processes but it reproduces a highly non-linear flow with a balance on the $\beta$-plane between steady wind forcing, non-linear bottom friction and inertial terms of the model. The model is similar to Veronis' model of wind-driven circulation in a rectangular basin (Veronis, 1966) but with a non-linear bottom friction.

The computation domain presents a rectangular basin with depth $H(=2 \mathrm{~km})$ centered at $35^{\circ} \mathrm{N}$. The domain is bounded by the rigid $(\Lambda)$ and open boundaries (Domain-A depicted in the top panel of Fig. 2). This basin extends $L_{2}=1000 \mathrm{~km}\left(L_{1}=1050 \mathrm{~km}\right)$ in the north-south (east-west) directions. A Cartesian coordinate system is used with the origin in the southwest corner. The $x_{1}$-axis points towards the east, and the $x_{2}$-axis towards the north.

The barotropic mode of the Princeton Ocean Model (POM) (Blumberg and Mellor, 1987) is applied to compute the horizontal velocities $u_{1}, u_{2}$, and the surface elevation $\zeta$ in Domain-A with no-slip boundary conditions. The circulation is forced by wind with stress varying with latitude:

$$
\frac{\tau_{\mathrm{w}}}{\rho_{0}}=\left(-10^{-3} \mathrm{~m}^{2} \mathrm{~s}^{-2}\right) \cos \frac{\pi x_{2}}{L_{2}},
$$

and the prescribed open boundary conditions $\left(u_{\mathrm{b}}\right.$ and $\left.\zeta_{\mathrm{b}}\right)$ explained below. Here, $\rho_{0}=1025.44 \mathrm{~kg} / \mathrm{m}^{3}$ is the reference density. The model runs with time step equaled to $2.5 \mathrm{~min}$ and reproduces the circulation with horizontal resolution of $50 \mathrm{~km}$.

The Coriolis parameter varies linearly with a $\beta$-plane approximation $f=f_{0}+\beta x_{2}$, where $f_{0}=2 \Omega \sin \varphi_{0}$ and $\beta=(2 \Omega / a) \cos \varphi_{0}$. Here, $\Omega$ and $a$ are the rate of rotation and the radius of the Earth, respectively; $\varphi_{0}=35^{\circ} ; f_{0}=0.73 \times 10^{-4} \mathrm{~s}^{-1}, \beta=2 \times 10^{-11} \mathrm{~m}^{-1} \mathrm{~s}^{-1}$. 
The horizontal kinematic viscosity is set to zero. The bottom stress is parameterized by the quadratic drag relation:

$$
\mathbf{w}=\left(\bar{w}_{1}, \bar{w}_{2}\right)=\left(\bar{\alpha} \rho_{0} \sqrt{u_{1}^{2}+u_{2}^{2}} u_{1}, \bar{\alpha} \rho_{0} \sqrt{u_{1}^{2}+u_{2}^{2}} u_{2}\right),
$$

where the drag coefficient $\bar{\alpha}=0.0025$. No model spin up exists for $\bar{\alpha}<0.0025$ when any solution is unstable.

The open boundary conditions are specified by Chu et al.'s (1997) approach. Accordingly to this approach POM is firstly integrated with wind stress (6a) and dissipation (6b) from rest $(u=v=0)$ and flat surface $(\zeta=0)$ for 150 days in a closed rectangular basin formed by extension of Domain-A up to $2000 \mathrm{~km}$ in the east (Domain-B shown in the bottom panel of Fig. 2). The velocity and surface elevation in the left part of Domain-B computed on day 10 are utilized as the initial conditions for the control run in Domain-A. The values of $u_{1}$ and $\zeta$ along the line $x_{1}=1050 \mathrm{~km}$ are the prescribed open boundary conditions $u_{\mathrm{b}}$ and $\zeta_{\mathrm{b}}$ between days 10 and 140 . The model spin up was found in Domain-A after 60-day integration. Here, the kinetic energy of the reference flow slowly decays with the power exponent of $1000 \mathrm{day}^{-1}$, oscillating with period of about 180 days.

The circulation pattern evolves from a single semi-closed gyre with a maximum velocity of about $0.35 \mathrm{~m} \mathrm{~s}^{-1}$ (Fig. 3a) and sea surface elevation between 0.05 and $0.1 \mathrm{~m}$ (not shown) to a multi-gyre structure with maximum velocities up to $0.9-1.0 \mathrm{~m} \mathrm{~s}^{-1}$ (Fig. 3b) and high surface elevation near $1 \mathrm{~m}$ in the west-northern part of the basin (not shown). This multi-gyre structure is a model spin up reached after 60-day integration.
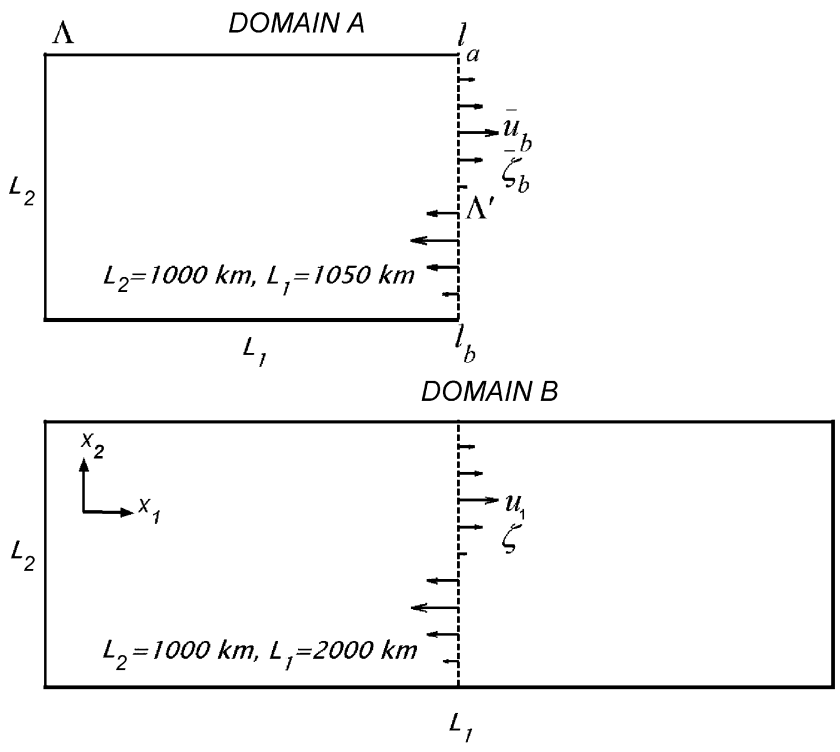

Fig. 2. Two areas for POM integration: Domain-A with the rigid $\Lambda$ (the boundary segment between $l_{\mathrm{b}}$ and $l_{\mathrm{a}}$ in the counter-clockwise direction) and open $\Lambda^{\prime}$ boundaries (top panel), and Domain-B with the rigid boundary (bottom panel). 

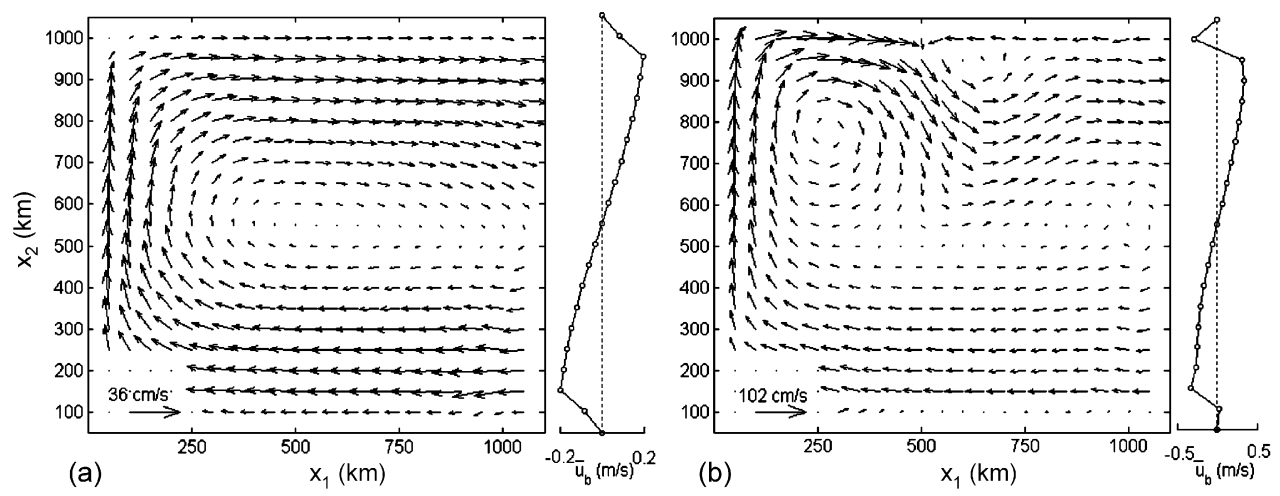

Fig. 3. The reference circulation and normal velocity $\bar{u}_{\mathrm{b}}$ on day 5 (a) and day 60 (b).

The model spin up is a non-linear regime identified by two non-dimensional numbers (Pedlosky, 1987):

$$
\operatorname{Ro}_{1}=\max \left(\frac{U}{f_{0} L}\right) \sim 0.7-0.1, \quad \operatorname{Ro}_{2}=\max \left(\frac{1}{f_{0} T}\right) \sim 0.12,
$$

where $U \sim 0.5-0.7 \mathrm{~m} \mathrm{~s}^{-1}$ is the characteristic velocity in the basin, $L \sim 10^{5} \mathrm{~m}$, and $T \sim 1$ day are the characteristic spatial and temporal scales of the flow, respectively.

The chosen model configuration allows us to use the same model for the analysis of model predictability affected by different sources of uncertainty: stochastic errors inserted in initial condition (the present study), wind (Ivanov and Chu, 2006), and open boundary conditions (Ivanov and $\mathrm{Chu}$, in preparation). Cross-correlations between different types of errors can also be studied.

Using the quasi-geostrophic approximation allows introducing a model phase space with the basis composed from $M$ eigenfunctions $\left[\psi_{1}, \ldots, \psi_{M}\right]$ of a plane Laplacian operator $\left(\Delta_{\perp}\right)$ defined in Domain-A (for details see Appendix A) and getting the following spectral representations for the reference solution:

$$
\hat{\Psi}(\mathbf{x}, t)=\sum_{m=1}^{M} \hat{A}_{m}(t) \psi_{m}(\mathbf{x})+\Psi_{\mathrm{harm}}(\mathbf{x}, t),
$$

and a perturbed solution

$$
\delta \Psi(\mathbf{x}, t)=\sum_{m=1}^{M} \delta A_{m}(t) \psi_{m}(\mathbf{x}),
$$

where $\delta \Psi=\hat{\Psi}-\Psi, \Psi$ is an individual forecast, and $\Psi_{\text {harm }}$ is the harmonic function described in Appendix A.

The spectral coefficients $\left[\hat{A}_{1}(t), \ldots, \hat{A}_{M}(t)\right]$ and $\left[\delta A_{1}(t), \ldots, \delta A_{M}(t)\right]$ define the reference and perturbed trajectories, starting, respectively, from points $\left[\hat{A}_{1}^{0}, \ldots, \hat{A}_{M}^{0}\right]$ and $\left[\delta A_{1}^{0}, \ldots, \delta A_{M}^{0}\right]$ in the phase space. Appendix A specifies the optimal truncation number for both the reference and perturbed flows as $M \approx 10^{2}$.

Therefore, the dimensionality of a dynamical system $(M)$ embedding the ocean model should be $\sim \mathrm{O}\left(10^{2}\right)$. However, the real number of degrees of freedom $\left(M_{0}\right)$ determined by 
Syrovich's approach (Syrovich, 1989; Aubry et al., 1991) is only about 15 (Chu and Ivanov, 2005).

We used a well-known classical stability analysis (Guckenheimer and Holmes, 1983) to classify the model spin up (quasi-equilibrium state) in dynamical system context. The governing equations in the spectral form (not shown) were linearized near the spin up state, and eigenvalues and eigenfunctions of appropriate tangent operator were calculated. The spectrum of the eigenvalues contained positive and negative real numbers only. Thus, the quasi-equilibrium state is an unstable focus in the model phase space. Any model trajectory tends to move toward (off from) this point in a phase plane determined by a pair of low-order (high-order) modes.

\section{Perturbations of initial conditions}

The main question in ensemble forecasting is how to generate a set of initial perturbations. The present study uses a Monte Carlo method to produce ensembles of perturbed solutions from running multiple POM simulations with initial perturbations, which are drawn randomly from a specified probability density function.

It is often assumed in ensemble forecasting that for a forecast model of nearly $10^{5}$ degrees of freedom, direct sampling of small size $\left[\sim \mathrm{O}\left(10^{2}\right)\right]$ ensembles is of limited utility because the inherent sampling error may overwhelm the desired covariance information. To correct these distortions different selective sampling procedures are used. See Palmer (2000) for extensive review of different techniques of ensemble generation including those based on singular (Molteni and Palmer, 1993) and breeding vectors (Toth and Kalnay, 1997). Miller and Ehret (2002) examined small ensembles drawn from spaces spanned by singular vectors and by bred vectors for non-linear dynamical systems with multiple attractors.

A sound approach for estimating model predictability was developed by Lermusiaux and Robinson (1999), Lermusiaux et al. (2000), and Lermusiaux (2001, 2002). The approach called the "error subspace statistical estimation" (Lermusiaux and Robinson, 1999) combines the dynamical equations for the ocean state in their discrete form in space, with a decomposition of error covariance to initialize and evolve the "dominant" eigendecomposition of the variability covariance matrix, merging data and dynamics. An essential feature of the dominant eigenvectors is that they indicate, evolve and organize the directions in the variable space that have largest statistical significance, based on a variance measure. Therefore, the approach provides a framework for investigation of large and complex system like the ocean. Lermusiaux et al. (2006) have demonstrated explicit capability of the approach through a number of practical examples including acoustical and biological models.

For a number of reasons we simply sample initial perturbations from specified, multivariate normal distributions. First, due to simplicity, our hydrodynamic model is able to produce forecast ensembles containing up to $\sim \mathrm{O}\left(10^{5}\right)$ perturbations. Such large ensembles have desirable statistical characteristics as revealed by a series of specialized statistical tests considered in Section 5 .

Second, the Latin Hypercube (LHC) design (Latin Hypercube, 2007) was applied to simulate a highly uniform distribution of an initial error in the phase space. Using pure probabilistic arguments, Downing et al. (1985) pointed out that the Latin Hypercube design is more effective than the classical Monte Carlo method. For obtaining dense error coverage with the same degree of homogeneity from Monte Carlo samples and through the LHC design $N^{M_{0}}$ and $N\left(2 M_{0}+2\right)$ statistical realizations are required, respectively. Here, $N$ is the number of statistical realizations necessary to simulate one degree of freedom. Typically, $N \sim \mathrm{O}\left(10^{3}\right)$. 
For $M_{0}=15$, the classical Monte Carlo method should require $\sim \mathrm{O}\left(10^{45}\right)$ initial perturbations for a statistically significant estimate. This is not feasible with available computer resources. Comparable results can be obtained by the LHC design approach with only $\sim \mathrm{O}\left(10^{4}\right)$ initial perturbations.

Third, our study focuses only on the physics of finite-amplitude errors and their contributions to losing model predictability. Thus, small forecast ensembles similar to those utilized by operational forecast models, are not considered here.

The initial perturbations $\mathbf{u}^{\prime}=\left[u_{1}^{\prime}(\mathbf{x}), u_{2}^{\prime}(\mathbf{x})\right]$ are assumed to be $2 \mathrm{D}$ isotropic Gaussian white noise (white noise-like perturbations (WNLPs)) with the two-point correlation function:

$$
\left\langle u_{i}^{\prime}(\mathbf{x}) u_{j}^{\prime}\left(\mathbf{x}^{\prime}\right)\right\rangle=I^{2} \delta_{i j} \delta\left(\mathbf{x}-\mathbf{x}^{\prime}\right),
$$

or 2D isotropic Gaussian spatially correlated noise (red noise-like perturbations (RNLPs)) with the two-point correlation function:

$$
\left\langle u_{i}^{\prime}(\mathbf{x}) u_{j}^{\prime}\left(\mathbf{x}^{\prime}\right)\right\rangle=I^{2} \delta_{i j} \exp \left[-\frac{\left(\mathbf{x}-\mathbf{x}^{\prime}\right)^{2}}{2 R_{\perp}^{2}}\right],
$$

where $\mathbf{x}=\left(x_{1}, x_{2}\right),\left(R_{\perp}, I^{2}\right)$ are correlation radius and noise variance (intensity of perturbations), respectively. These perturbations are directly added to the initial conditions. The technical details of generating Gaussian noises with correlation functions (9) and (10) can be found in Sabel'feld (1991). The non-dimensional intensity of the initial perturbations $\bar{I}^{2}=I^{2} / I_{0}^{2}\left(I_{0}^{2}=1 \mathrm{~m}^{2} \mathrm{~s}^{-2}\right)$ will be used below.

Noise with correlation functions (9) and (10) is the popular model of errors for optimal interpolation or spline fitting. Both of these procedures are applied to construct initial conditions for ocean models from irregularly spaced data (Brasseur et al., 1996; Robinson et al., 1996 and others).

\section{Optimal ensemble size}

The LHC design approach provides the dense error coverage of the model phase space for $\sim \mathrm{O}\left(10^{4}\right)$ initial perturbations. This number of initial perturbations is a trade-off between the ensemble ability to reproduce main features of PE statistics, and the computational cost. However, the optimal number of initial perturbations $\left(N_{\text {opt }}\right)$ should be specified for the concrete ocean model. We suggest to estimate this number through the Kullback-Leibler (KL) distance (the relative entropy) (White, 1994).

The KL distance is a natural distance function from a "true" probability density, $F_{\infty}$, to a "target" probability density, $F_{N}$. For continuous density functions, the KL distance is defined as

$$
\mathrm{KL}_{N}\left(F_{\infty} \mid F_{N}\right)=\int_{0}^{\infty} \mathrm{d} \tau F_{N}(\tau) \log \left[\frac{F_{\infty}(\tau)}{F_{N}(\tau)}\right],
$$

where $F_{N}(\tau)$ and $F_{\infty}(\tau)$ are $\tau$-PDFs computed for an $N$ sample ensemble and a hypothetic ensemble with infinite sampling, respectively. In practice, a difference between two distributions is negligible if $\mathrm{KL}_{N} \leq 5.0 \times 10^{-3}$ (White, 1994).

To calculate the KL distance we suppose $F_{\infty}=F_{100,000}$ because only small differences in $\tau$ statistics estimated from ensembles of $5.0 \times 10^{3}, 1.0 \times 10^{4}, 2.0 \times 10^{4}, 5.0 \times 10^{4}$ and $1.0 \times 10^{5}$ samples have been observed. Typical behavior of $\mathrm{KL}_{N}$ with the growth of $N$ is shown in Fig. 4a. 

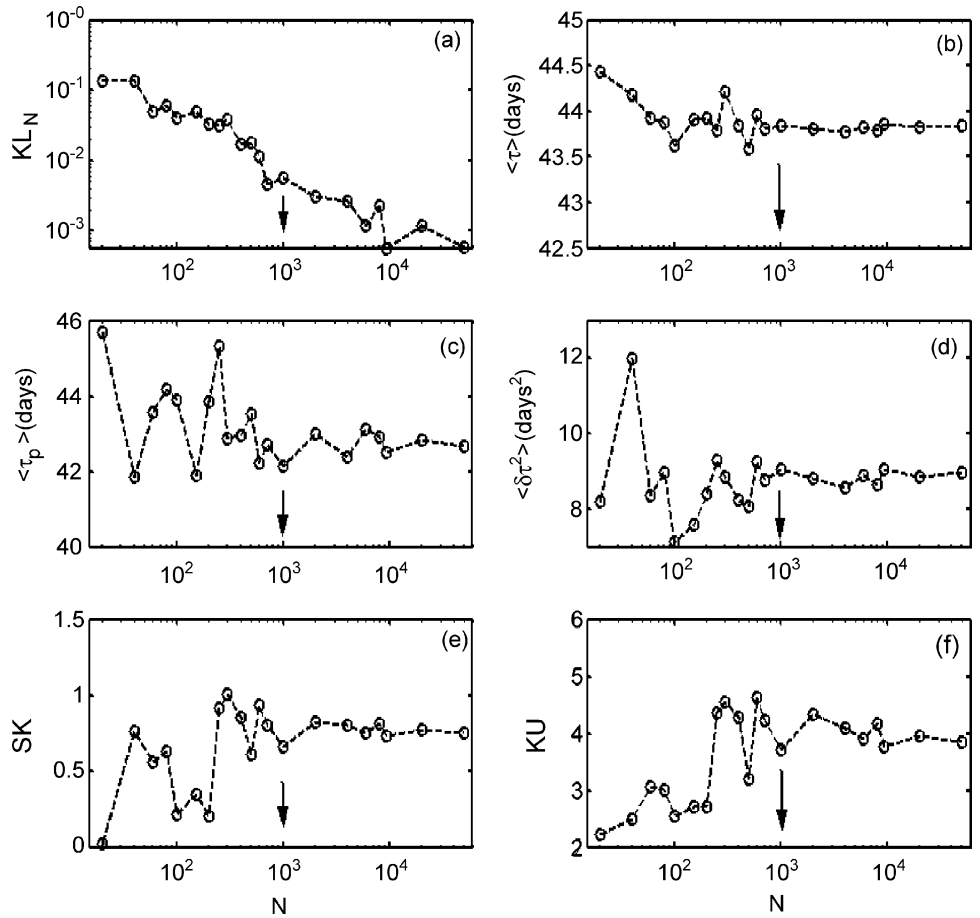

Fig. 4. Sensitivity of $\tau$-statistics to the ensemble size (N): (a) the Kullback-Leibler distance; (b) the $\tau$-mean; (c) the $\tau$-most probable; (d) the $\tau$-variance; (e) the $\tau$-skewness; (f) the $\tau$-kurtosis. Initial perturbations are red noise with the correlation radius $R_{\perp}=112 \mathrm{~km}$ and the intensity $\bar{I}^{2}=0.1, \bar{\varepsilon}^{2}=0.2$. Arrows indicate $N_{\text {opt }}=10^{3}$.

The $\mathrm{KL}_{N}$ rapidly reduces with $N$ from $2.0 \times 10^{-1}(N=20)$ to $4.0 \times 10^{-3}\left(N=10^{3}\right)$. Therefore, $N_{\text {opt }}$ was chosen as $10^{3}$.

The first four moments of IPT also quickly converge as $N$ increases to $10^{3}: \tau$-mean to 43.8 days (Fig. 4b), $\tau$-most probable to 42.6 days (Fig. $4 \mathrm{c}$ ), $\tau$-variance to 8.8 days $^{2}$ (Fig. $4 \mathrm{~d}$ ), and $\tau$-skewness to 0.75 day (Fig. 4e). Kurtosis is most sensitive to the choice of $N$ (Fig. 4f), and varies between 3.9 and 4.1 for $N \sim 10^{3}$. However, these variations are quite small to mask the non-Gaussian feature of IPT statistics (Gaussian statistics is identified by both $\mathrm{SK}=0.0$ and $\mathrm{KU}=3.0$ ).

By varying characteristics of initial perturbations $\left(\bar{I}^{2}\right.$ and $\left.R_{\perp}^{2}\right)$ we have found that $N_{\text {opt }}=10^{3}$ is an acceptable choice for any combinations of $\bar{I}^{2}$ and $R_{\perp}^{2}$. Thus, all forecast ensembles below have large size $\left(N_{\text {opt }}=10^{3}\right)$ and are not rank-deficient $\left(N_{\text {opt }} \gg M_{0}\right)$. This reduces the sampling errors significantly.

\section{Prediction error evolution}

\subsection{Different stages of PE evolution}

A number of stages for PE evolution (at least four) are observed for both WNLPs (Fig. 5a) and RNLPs (Fig. 5b). All these stages are clearly identified by the growth rate $Q=\mathrm{d}(\ln \langle J\rangle) / \mathrm{d} t($ Fig. $6 \mathrm{a}$ and $b$ ). 

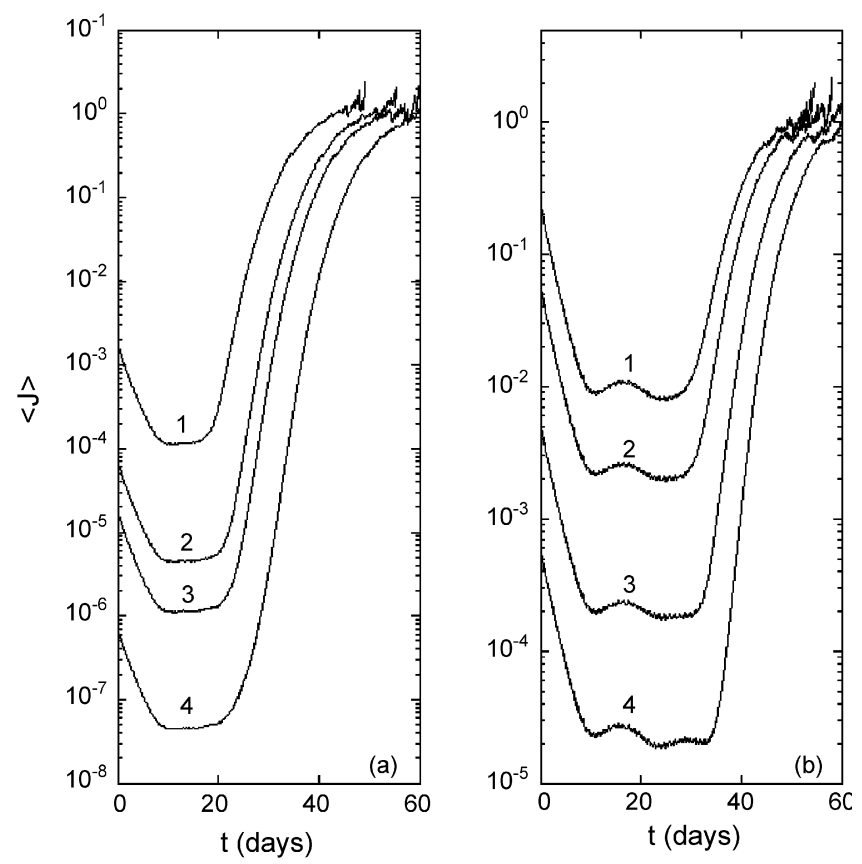

Fig. 5. Temporal evolution of the root mean square error $\langle J\rangle$ for various initial perturbations-(a) WNLPs with different noise intensities $\left(\bar{I}^{2}\right)$ : 0.05, 0.01, 0.005, and 0.001 (denoted by $\left.1,2,3,4\right)$ and (b) RNLPs with the correlation radius $\left(R_{\perp}\right)$ of $70 \mathrm{~km}$ and different noise intensities $\left(\bar{I}^{2}\right): 0.02,0.01,0.003$ and 0.001 (denoted by 1, 2, 3, 4).
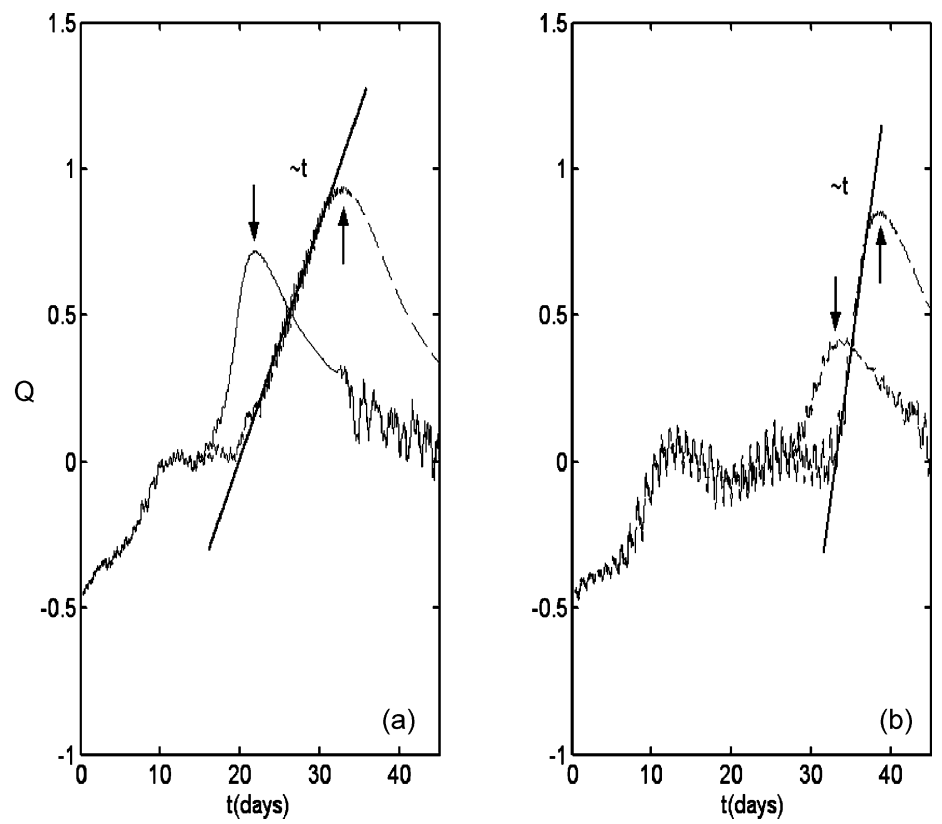

Fig. 6. The growth rate $Q$ for (a) WNLPs and (b) RNLPs with the correlation radius $\left(R_{\perp}\right)$ of $70 \mathrm{~km}$. The solid and dashed curves correspond to $\bar{I}^{2}=1.0 \times 10^{-3}$ and 0.5 . Black arrows indicate boundaries between the linear and non-linear predictability regimes for different characteristics of initial perturbations. 
Initial error decay is observed for the first 10 days of PE evolution (Fig. 5a and b) where the growth rate $Q$ evolves as

$$
Q \approx Q_{0}\left[\exp \left(\alpha_{0} t\right)-\alpha_{1}\right]
$$

where $\alpha_{0}$ is the decay exponent, $Q_{0} \alpha_{1} \approx-0.45, \alpha_{0}=\ln \alpha_{1} / t_{1}, t_{1}=10$ days.

Non-exponential initial error decay corresponding to (12) differs from a quasi-exponential decay obtained in Wirth and Ghil's (2000) model with the dissipative operator $\nu \Delta_{\perp}$ and quite large horizontal viscosity $v$. In contrast to this model the zero horizontal viscosity is used in our model. Therefore, the non-exponential decay seems to be caused by non-linear bottom friction.

Then $\langle J(t)\rangle$ retains quasi-stationary low values during 10 and 20 days for WNLPs (Fig. 5a) and RNLPs (Fig. 5b), respectively. At this stage (stagnation stage) the growth rate $Q$ oscillates near zero (Fig. 6a and b).

During the third stage (after days 20 and 30 for WNLPs and RNLPs, respectively) PE grows faster than exponentially (Fig. 5a and b). Fig. 6a and b shows that here the growth rate increases with $t$ linearly:

$$
Q \sim t
$$

Mechanisms for PE to grow faster than (quasi)-exponentially, were discussed earlier in the scientific literature (Lacarra and Talagrand, 1988; Smith et al., 1999 among others). Therefore, they are not in the focus of the present study. Duration of "super-exponential" error growth does not exceed 7-10 days.

Non-linear interactions among various scales slow down (Fig. 6a and b), and then, limit further growth of PE (Fig. 5a and b). The transition from the linear to non-linear predictability regime is identified by a behavior of the growth ratio: $Q$ reaches a maximum value and then quickly reduces. Although linear effects can also stimulate similar behavior of $Q$ (Smith et al., 1999), more detailed analysis provided in Section 7 gives evidence for the non-linear predictability regime.

The duration of the non-linear predictability regime (up to 10-15 days) does not seem to be short, and is comparable with the time when the PE grew faster than exponentially. The larger the error amplitude, the faster the transition from linear to non-linear predictability regime. Therefore, the non-linear regime should play an important role in understanding model predictability and its limits.

\subsection{Initial error decay}

Initial error decay was documented earlier by Lorenz (1996), Brasseur et al. (1996), Molteni et al. (1996), Wirth and Ghil (2000), Vannitsem and Nicolis (1997), Snyder et al. (2003) and others. Their results show (a) the relation of the decay to model dissipation, and (b) that stability of the reference solution estimated by the kinetic energy norm is less than when other norms are used.

The initial error decay quantified by the kinetic energy norm diminishes or even disappears with reducing the coefficient of horizontal viscosity $v_{\mathrm{h}}$, when dissipation processes were parameterized by a hyper-dissipation operator $-v_{\mathrm{h}} \Delta_{\perp}^{2}$ (Snyder et al., 2003; Vannitsem and Nicolis, 1997).

In contrast to this, Wirth and Ghil (2000) have demonstrated that the square root of the streamfunction error variance $(J)$ for randomly inserted small-amplitude perturbations first decays due to viscous damping parameterized by the usual dissipative operator $v \Delta_{\perp}$. Although for smaller values of horizontal viscosity $v$ the decay was slower, it did not disappear with reducing $v$. 
Our computations demonstrate the existence of the initial error decay for both the kinetic energy norm and norm (1) only due to non-linear bottom friction if the friction coefficient $\alpha \geq \bar{\alpha}$. The choice of $J_{\text {norm }}=1$ in norm (1) does not change the obtained result, although the decay becomes slower. The decay also exists for non-zero coefficients $\left(v\right.$ and $\left.\nu_{\mathrm{h}}\right)$ of horizontal viscosity.

Since the initial error decay, in general, does not depend on the amplitude of initial perturbations (Fig. 5a and b), to understand its physics we limit ourselves to the case of small-amplitude initial perturbations. In our opinion, the initial error decay may be explained by features of a non-linear dissipation scheme and statistical features of initial perturbations with correlation functions (9) and (10).

Using the assumption on small amplitudes of initial perturbations, the term, which is responsible for PE dissipation (see Appendix B), can be written as

$$
\begin{aligned}
& \left\langle F_{1} u_{1}^{\prime}\right\rangle \approx \alpha E^{-1 / 2}\left[\gamma_{1}\left\langle\left(u_{1}^{\prime}\right)^{2}\right\rangle+\gamma_{2}\left\langle u_{1}^{\prime} u_{2}^{\prime}\right\rangle\right], \\
& \left\langle F_{2} u_{2}^{\prime}\right\rangle \approx \alpha E^{-1 / 2}\left[\gamma_{3}\left\langle\left(u_{2}^{\prime}\right)^{2}\right\rangle+\gamma_{2}\left\langle u_{1}^{\prime} u_{2}^{\prime}\right\rangle\right],
\end{aligned}
$$

where $\left(\bar{u}_{1}, \bar{u}_{2}\right)$ and $\left(u_{1}^{\prime}, u_{2}^{\prime}\right)$ are velocities of the reference and perturbed solutions, respectively; $E=\bar{u}_{1}^{2}+\bar{u}_{2}^{2}$ is the kinetic energy of the reference flow, $\gamma_{1}=2 \bar{u}_{1}^{2}+\bar{u}_{2}^{2}, \gamma_{2}=\bar{u}_{1} \bar{u}_{2}$ and $\gamma_{3}=$ $\bar{u}_{1}^{2}+2 \bar{u}_{2}^{2}$.

During the first stage (initial error decay), the PE dissipation is only determined by the first terms in the right-hand sides of Eqs. (14) and (15), because the initial perturbations are statistically isotropic: $\left\langle u_{i}^{\prime}(\mathbf{x}) u_{j}^{\prime}(\mathbf{x})\right\rangle=0$, if $i \neq j$ (the isotropy feature). Both renormalized coefficients of friction $\alpha E^{-1 / 2} \gamma_{1}$ and $\alpha E^{-1 / 2} \gamma_{2}$ are positive and grow with increasing kinetic energy as $E^{1 / 2}$.

During the stagnation stage (Fig. 5a and b), the correlations between $u_{1}^{\prime}$ and $u_{2}^{\prime}$ become not small. The growth of the renormalized coefficients is compensated by the term $\gamma_{2}\left\langle u_{1}^{\prime} u_{2}^{\prime}\right\rangle \leq 0$. This process stops the PE decay. After day 20 for WNLPs and day 30 for RNLPs, $\gamma_{2}\left\langle u_{1}^{\prime} u_{2}^{\prime}\right\rangle \leq 0$ continues to reduce the dissipative terms $\left\langle F_{1} u_{1}^{\prime}\right\rangle$ and $\left\langle F_{2} u_{2}^{\prime}\right\rangle$, what reduces the model dissipation and stimulates the growth of prediction error.

Non-linear bottom friction (6b) leads to effective decay of the prediction error at all spatial scales larger than a scale determined by the 100th mode (see Fig. 7a and b). At this stage of PE evolution geostrophic adjustment inducing an upscale flux of the prediction error does not play an important role. A measure of re-distribution of the kinetic energy among modes is the spectral entropy (Aubry et al., 1991). The entropy is equaled to 1 when the kinetic energy is homogeneously distributed among all the modes (maximum disorder), and to 0 when the energy is contained in a single mode (maximum order). The spectral entropy is computed by

$$
S=-\left[\log \left(M-m_{0}\right)\right]^{-1} \sum_{m=m_{0}}^{M} p_{m} \log p_{m}, \quad p_{m}=\frac{b_{m}^{2}}{b}, \quad b=\sum_{m=m_{0}}^{M} b_{m}^{2},
$$

where $b_{m}$ are $\hat{A}_{m}\left(\delta A_{m}\right)$ for the reference (the perturbed) solution; $m_{0}$ and $M$ determine the spectral band for calculating the spectral entropy.

The spectral entropy computed for the PE (the reference solution) is shown in Fig. 8a (b). Fig. 8a does not show any significant re-distribution of kinetic energy among different flow scales up to 10-12 days. A strong upscale flux of PE is observed for low-order modes (from 1 to 20) after day 12: the spectral entropy approximately reduces into half. Such a process seems to be considerably weaker for high-order modes. In contrast to the PE, the complexity of the reference flow monotonically grows up to day 40 (Fig. 8b). 

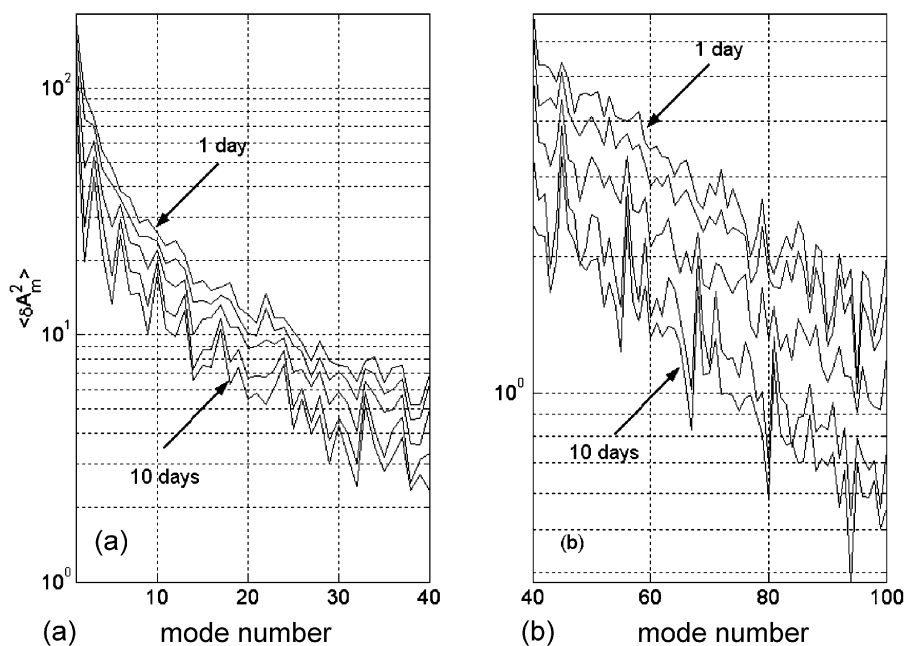

Fig. 7. Spectra of $\mathrm{PE}\left\langle\delta A_{m}^{2}\right\rangle$ at large $(m=1-40)$ and small $(m=40-100)$ scales between days 1 and 10 . The initial error is the WNLP with $\bar{I}^{2}=0.1, \bar{\varepsilon}^{2}=0.5$.
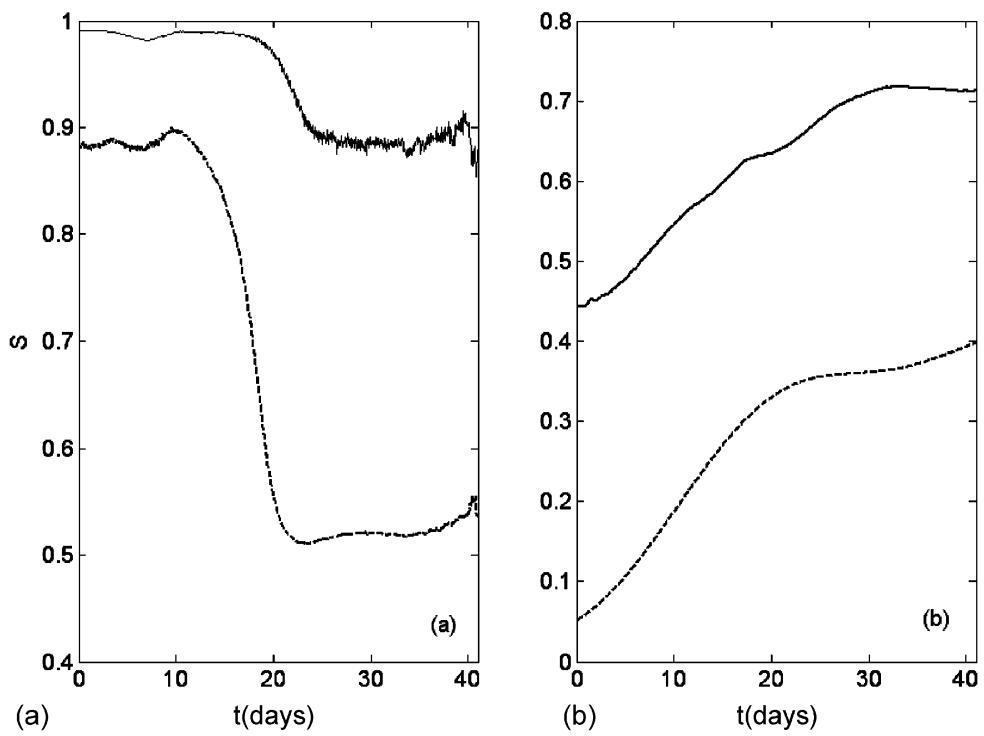

Fig. 8. The spectral entropy $S$ of (a) PE and (b) the reference solution. Dashed and solid lines are computations for the first $20\left(m_{0}=1, M=20\right)$ modes and last $50\left(m_{0}=50, M=100\right)$ modes, respectively. The initial error is the WNLP with $\bar{I}^{2}=0.1, \bar{\varepsilon}^{2}=0.5$.

\section{Response to finite-amplitude initial perturbations}

Linear intuition suggests that the larger the amplitude of the initial perturbations, the higher the probability of obtaining low model predictability. Prediction errors should steadily increase with a prediction time scale in the linear predictability regime. In contrast to this, forecasts skill may decay slower when amplitude of initial perturbations grows. Our computations show that the 
growing perturbations rapidly adopt a horizontal scale comparable to that of the reference state (linear predictability regime), and their further growth is limited by interactions with this state and among them (non-linear predictability regime). In the non-linear predictability regime the PE demonstrates clear contributions from the cumulative effects of flow scales, and the predictability time is no longer measured by the inverse of the leading Lyapunov exponent. Moreover, model predictability enhances with the growth of the correlation radius $R_{\perp}$ and is less sensitive to the choice of the intensity $\bar{I}^{2}$.

To understand correlations between model predictability, the amplitude of initial perturbations, the correlation radius and the noise intensity, let us show how the amplitude of initial perturbations is affected by $R_{\perp}$ and $\bar{I}^{2}$. Using the spectral representation for noise covariance matrix obtained in Appendix $\mathrm{C}$, the maximum amplitude of initial perturbations is estimated as

$$
\left\langle\left(\delta A_{\max }^{0}\right)^{2}\right\rangle^{1 / 2}=(2 \pi)^{1 / 2} L_{1} L_{2} R_{\perp}^{2} \bar{I} \operatorname{erf}\left(\frac{L_{1}}{\sqrt{2} R_{\perp}}\right)^{1 / 2} \operatorname{erf}\left(\frac{L_{2}}{\sqrt{2} R_{\perp}}\right)^{1 / 2} \lambda_{\max }^{1 / 2},
$$

where $\lambda_{\max }$ is the maximum eigenvalue of a matrix determined in Appendix C.

Eq. (17) clearly shows that the amplitude of initial perturbations is most sensitive to the correlation radius but not the noise intensity, because the amplitude may grow as $R_{\perp}^{2}$ and only linearly with $\bar{I}$.

Our computations demonstrate the existence of the linear predictability regime identified by non-Gaussian statistics and (quasi)-exponential growth of PE, for a small correlation radius $\left(R_{\perp} \ll 50 \mathrm{~km}\right.$, the case of WNLPs) and large noise intensities $\left(\bar{I}^{2} \leq 0.2\right)$. For WNLPs, typical $\tau$-PDF was close to Gaussian if $\bar{I}^{2} \sim 0.01-0.05$. The growth of $\bar{I}^{2}$ up to $0.1-0.2$ resulted in a weak asymmetry for the $\tau$-PDF $(\mathrm{SK} \rightarrow 0.15)$, and departs from non-Gaussian (KU $\rightarrow 3.10$ ). However, although such a $\tau$-PDF has a short tail (labeled by 1 in Fig. 9a), it was still close to Gaussian and the mean $\tau$-IPT reduced with the growth of amplitude of initial perturbations. The
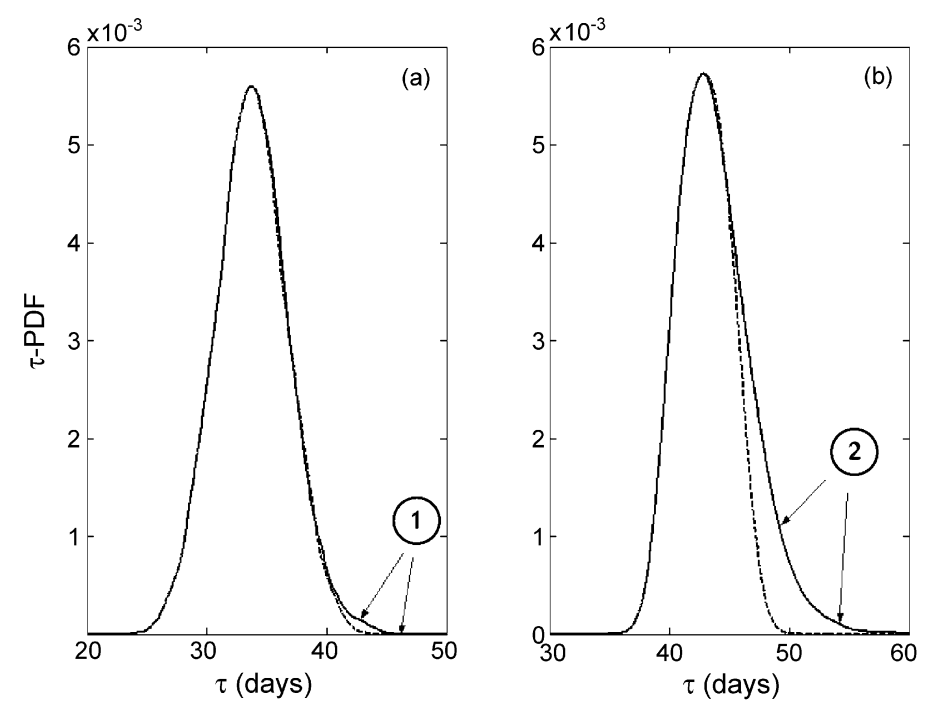

Fig. 9. $\tau$-PDFs for (a) WNLPs with $\bar{I}^{2}=0.1$ and $\bar{\varepsilon}^{2}=0.5$, and (b) RNLPs with $\bar{I}^{2}=0.1$ and $R_{\perp}=125 \mathrm{~km}$, and $\bar{\varepsilon}^{2}=0.2$. Skewness and kurtosis are 0.15 and 3.09 in case (a), and 0.77 and 3.95 in case (b). Dashed lines indicate mirror reflections of the left-hand side tails of $\tau$-PDFs. 

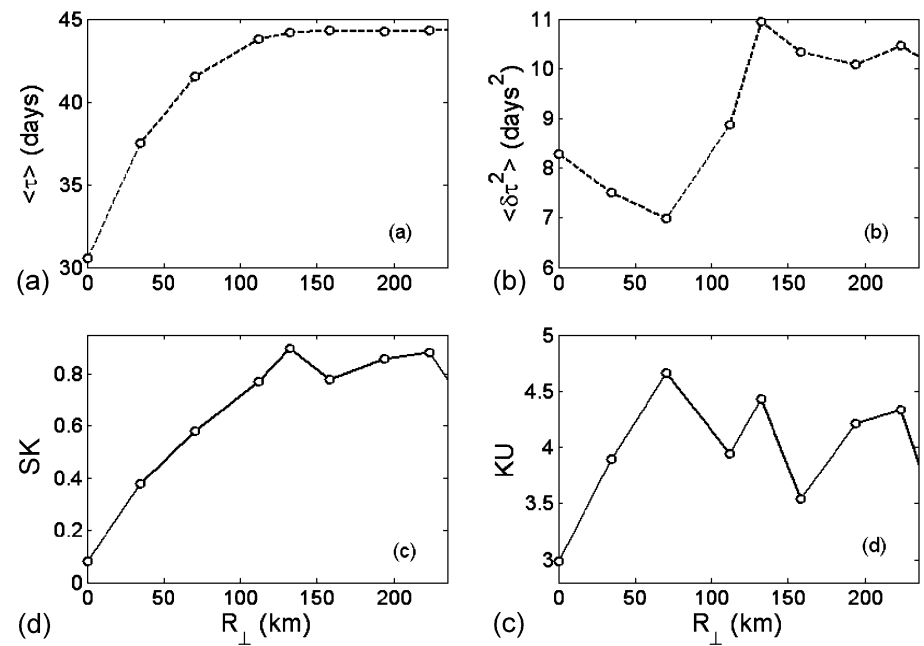

Fig. 10. Dependence of $\tau$-statistics on the correlation radius $R_{\perp}$ for RNLPs with the noise intensity $\bar{I}^{2}=0.1$, and $\bar{\varepsilon}^{2}=0.2$. (a) $\tau$-mean, (b) $\tau$-variance, (c) $\tau$-skewness, and (d) $\tau$-kurtosis.

$\tau$-PDF quickly departs from Gaussian with the growth of $\bar{I}^{2}$ after 0.2 . However, from the physical point of view, such initial perturbations seem to be too large to exist in reality.

The non-linear predictability regime appears as $R_{\perp}$ grows. Both highly non-Gaussian $\tau$-PDFs, and the mean IPT that grows with $R_{\perp}$, indicate that the PE becomes non-linear. A typical nonGaussian $\tau$-PDF ( $\mathrm{SK} \approx 0.8, \mathrm{KU} \approx 4.0$ ) computed for a finite correlation radius is demonstrated in Fig. 9b. The long PDF tail (labeled by 2 in Fig. 9b) is clearly seen in this figure. The tail is formed by rare individual forecasts (IPT up to 60 days), each of which is longer than the mean ensemble forecasting (IPT of about 44 days).

Asymmetry of $\tau$-PDFs becomes higher for the larger values of correlation radius $R_{\perp}$ (Fig. 10c). $\mathrm{SK}$, which is a measure of asymmetry, increases up to 0.8 when $R_{\perp}$ tends to $100 \mathrm{~km}$. Larger values of mean IPT (Fig. 10a) and $\tau$-variances (Fig. 10b) correspond to more asymmetric PDFs. Highly non-Gaussian $(\mathrm{KU} \approx 4$, Fig. $10 \mathrm{~d})$ and sharp $\tau$-PDFs with long tails stretching to large prediction times accompany this non-linear predictability regime. The explicit growth of mean predictability time observed with the growth of correlation radius $R_{\perp}$ is a strong evidence of the non-linear predictability regimes caused by inhomogeneous morphology of the model phase space (Kaneko, 1998).

\section{Weibull statistics}

The results above demonstrated a fast departure of $\tau$-PDFs from Gaussian shape with the growth of amplitude of initial perturbations. For finite-amplitude initial perturbations $\tau$-PDFs were highly non-Gaussian and asymmetric. The following question arises: what distribution is the best fit for such $\tau$-PDFs? If the analytical form of such a distribution can be found, it can be useful for the parametric estimate of ensemble generated PDFs from limited observation samples and small forecast ensembles.

Our computations demonstrate that the tailed $\tau$-PDFs reconstructed by a non-parametrical technique based on the Epanichenikov's kernel and the bootstrap re-sampling procedure (Good, 
1996) directly from the Monte Carlo samples, are fitted by the three-parameter Weibull distribution function (Bury, 1999):

$$
f(\tau)=\frac{\beta}{\eta}\left(\frac{\tau-\gamma}{\eta}\right)^{\beta-1} \exp \left[-\left(\frac{\tau-\gamma}{\eta}\right)^{\beta}\right] .
$$

Here, $\eta, \gamma$ and $\beta$ are scale, shape and location parameters. We found no difference between the reconstructed PDFs and their Weibull counterparts with at least a 95\% confidence level.

We suggest to identify the distribution parameters of (18) by the probability weighted moments (PWMs). Definition of PWMs and their features are given in Greenwood et al. (1979). For a number of reasons these moments seem to be more attractive for the practical calculations of prediction scales than the classical statistical moments (CSMs). First, the PWMs are less sensitive to sampling than the CSMs. Second, the classical statistical moments may not exist for PDFs with long (heavy) tails. For example, if a cumulative distribution function $P$ has asymptotic tail $\sim t^{-\sigma}$ as $t \rightarrow \infty$, then the classical $\tau$-moments of $(k-\sigma-1)$ order do not exist because integral (5) does not converge. In contrast to the CSMs, the PWMs exist for any tailed PDFs.

From the physical point of view, the model predictability is also clearly quantified by $\tau$-CDF, which is the probability that a model is able to predict with accuracy higher than $\bar{\varepsilon}$, at times larger than $t$ (see, also Section 1). Accordingly to Bury (1999) CDF for Weibull distribution (18) can be written as

$$
P(t)=\exp \left[-\left(\frac{t-\gamma}{\eta}\right)^{\beta}\right]
$$

The parameter $\beta$ is a measure of how quickly the $\tau$-CDF decays with $t$. The super-exponential $(\beta>1)$, exponential $(\beta=1)$ and sub-exponential $(\beta<1)$ decay regimes correspond to small, intermediate and high probabilities to obtain the enhanced predictability in an individual forecast.

The following method was used to estimate distribution parameters of (18) from ensemble samples.

The PWMs can be estimated in two ways: from Eq. (D1), using analytical representation (19), and directly from ensemble samples through Eq. (D2). The distribution parameters are obtained through a misfit between these estimations. For details see Appendix D.

Typical $\tau$-CDFs computed for both WNLPs and RNLPs are shown in Fig. 11a and b, respectively. A more tailed CDF corresponds to the RNLPs (compare Fig. 11a and b). That indicates a higher probability of long individual forecasting for spatially correlated initial errors than for WNLPs. If parameters for distribution (18) are known, there is a chance to predict an asymptotic behavior of $\tau$-CDF tail with $t$, and understand contributions of rare long forecasts to model predictability.

The last statement is illustrated through a simple example. Let us estimate a contribution of rare long forecasts to the growth of $\tau$-mean with $R_{\perp}$. The mean IPT calculated for WNLPs $\left(\bar{I}^{2}=0.1\right)$ added to initial conditions, was equal to about 30.41 days, when the tolerance level was $\bar{\varepsilon}^{2}=0.2$. For initial RNLPs with the same intensity but the correlation radius $R_{\perp}=125 \mathrm{~km}$, the Weibull distribution parameters are estimated as

$$
\gamma \approx 37.5 \text { days, } \quad \eta \approx 6.2 \text { days, } \quad \text { and } \quad \beta \approx 2.1 \text {. }
$$



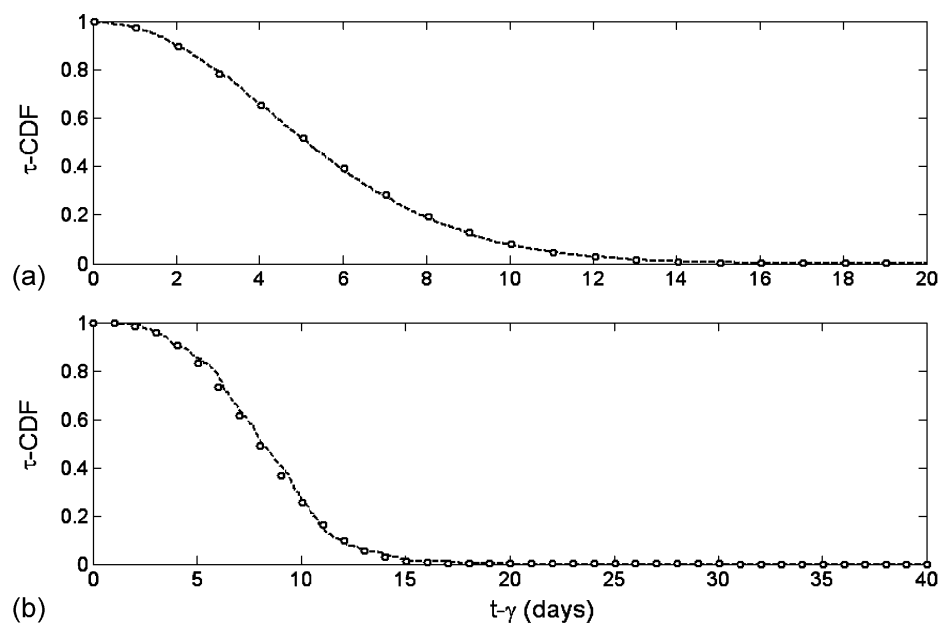

Fig. 11. $\tau$-CDF computed for (a) WNLPs with $\bar{I}^{2}=0.1$ and (b) RNLPs with $\bar{I}^{2}=0.1$ and $R_{\perp}=70 \mathrm{~km}$. In both these cases $\bar{\varepsilon}^{2}=0.1$. Here, the circles and dashed lines represent $\tau$-CDFs computed directly from the Monte Carlo samples and by the probable weighted moment technique, respectively. The location parameter $\gamma$ equals to 20.4 days in case (a) and 38.3 days in case (b).

For Weibull distribution (18) the $\tau$-mean is calculated as

$$
\langle\tau\rangle=\gamma+\eta \Gamma\left(1+\frac{1}{\beta}\right),
$$

where $\Gamma$ is the gamma function.

Substituting parameters (20) into Eq. (21) yields,

$$
\langle\tau\rangle \approx 44.2 \text { days. }
$$

Therefore, the mean IPT has grown on 13.8 days. The tail lengthening stimulates approximately $50 \%$ ( $\eta \approx 6.2$ days) of this growth. The other half of the growth estimated as $\gamma-\langle\tau\rangle=7.1$ days is caused by variations of the location parameter.

\section{Transition from linear to non-linear predictability regime}

The above results showed the existence of two predictability regimes. From the practical point of view it is important to understand how quickly the linear predictability decays and what is a threshold $\delta$ on amplitude of initial perturbations, above which the non-linear predictability regime dominates. There are two approaches for determining the duration of the linear predictability regime. One is to compare the evolution of a perturbation under the full non-linear model with its evolution under the tangent linear model in order to quantify the PE in this model as a function of time (for example see Vukicevic, 1991).

Another approach is to develop a criterion. For example Gilmour et al. (2001) suggested estimating duration of the linear predictability regime by monitoring the evolution of twin perturbations under the full non-linear model. Our idea is to demonstrate that the decay of the linear predictability regime correlates with the changes in behavior of $\tau$-variance. 

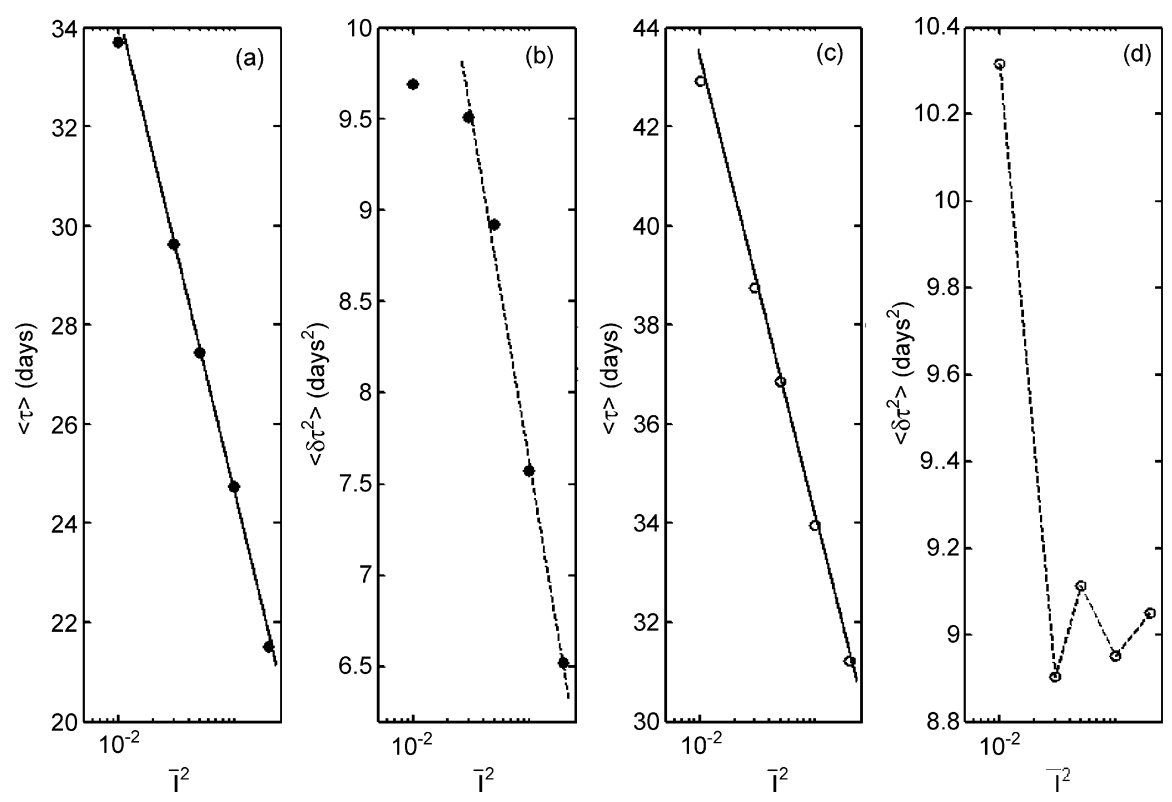

Fig. 12. Dependence of $\tau$-statistics on the noise intensity for WNLPs: (a) and (b) for $\bar{\varepsilon}^{2}=0.01$; (c) and (d) for $\bar{\varepsilon}^{2}=0.5$. Logarithmic law (23) is indicated by solid line in (a) and (c). Dashed line in (b) corresponds to logarithmic law (24).

Let us introduce the non-dimensional parameter $\mu=\bar{\varepsilon}^{2} / \bar{I}^{2}$ that is a measure for degree of smallness of initial perturbations, and estimate it for a number of combinations of $\bar{\varepsilon}^{2}$ and $\bar{I}^{2}$. The linear predictability regime occurs for $2.5 \times 10^{-3}<\mu \leq 1.0$ (Fig. 12a and b) when $\tau$-mean and $\tau$-variance evolve along the following logarithmic laws:

$$
\begin{aligned}
& \langle\tau\rangle \sim \ln \left(\frac{\bar{\varepsilon}^{2}}{\bar{I}^{2}}\right), \\
& \left\langle\delta \tau^{2}\right\rangle \sim \ln \left(\frac{\bar{\varepsilon}^{2}}{\bar{I}^{2}}\right) .
\end{aligned}
$$

Eq. (23) coincides with the well-known law of the exponential growth of infinitesimal initial perturbations on a chaotic attractor (Lorenz, 1996). Logarithmic law (24) was theoretically predicted by Chu et al. (2002).

The departure from the linear predictability is observed for $1.0<\mu \leq 16.7$ (Fig. 12c and d). Although, here, the $\tau$-mean still follows the (quasi)-logarithmic law (23) with the growth (reduction) of $\mu\left(\bar{I}^{2}\right)$, and deviations from this law do not seem to be essential for small $\bar{I}^{2}$ and large $\bar{\varepsilon}^{2}$, the $\tau$-variance does not demonstrate a behavior that Eq. (24) predicts. It is practically constant (about 9 days $^{2}$ ) for $\bar{I}^{2}$ varied between $0.5 \times 10^{-1}$ and 0.2 (Fig. 12d). That shows no correlations between the $\tau$-mean (ensemble mean) and $\tau$-variance (ensemble spread) when the amplitude of initial perturbations exceeds the threshold $\mu \approx 1.0$.

The intermediate domain between the linear and non-linear predictability regimes, when there are no correlations between ensemble mean and spread, is also characterized by small changes in 
scale and shape parameters $(\eta, \beta)$ estimated as

$$
\eta \approx 9.6 \pm 0.1, \quad \beta \approx 3.4 \pm 0.1 .
$$

Here, $\tau$-statistics is still close to Gaussian because the skewness and the kurtosis are close to $0(|\mathrm{SK}|<0.5)$ and $3\left(|\mathrm{KU}-3.0|<1.5 \times 10^{-1}\right)$, respectively.

The lack of statistically significant correlations between the forecast skill and ensemble spread is sometimes observed in ensemble modeling for the atmosphere and ocean. For example, Moore (1999) performed a series of experiments, in which he applied different methods of ensemble generation to a quasi-geostrophic model of the Gulf Stream. He obtained no statistically significant relationships between forecast skill and ensemble spread in a number of cases and explained them only by poor statistics in the forecast experiments. Our computations show that the same effects can appear when the initial perturbations have quite large amplitudes.

\section{Predictability horizon}

Asymptotic behavior of the $\tau$-CDF with time determines the predictability horizon for the hydrodynamic model used here. Model predictability horizon is defined as the maximum prediction time $\left(\tau_{\text {hor }}\right)$ for the given model and statistics of initial perturbations (Kravtsov, 1993) and can be calculated by

$$
\tau_{\text {hor }}=\gamma+\eta\left[-\ln \bar{P}^{*}\right]^{1 / \beta},
$$

where $\bar{P}^{*}$ is the probability that $\tau_{\text {hor }}$ will be achieved in an individual forecast.

Let us estimate the model predictability horizon for the example discussed in Section 8. Substituting parameters (20) into Eq. (26) yields,

$$
\tau_{\text {hor }} \approx 50.21,52.79 \text { and } 55.03 \text { days }
$$

for $\bar{P}^{*}=0.01,0.001$ and 0.0001 , respectively. Thus, for the RNLPs with the fixed values of $R_{\perp}$ and $\bar{I}^{2}$, and $\bar{\varepsilon}^{2}=0.20$, the model predictability horizon is limited to 52-55 days and any individual forecast longer than 55 days, is improbable.

\section{Conclusions}

We have used a simple shallow-water model to understand predictability of perfect ocean models. This model is a highly idealized representation of some aspects of the ocean dynamics and naturally, cannot simulate re-distributions of PE between barotropic and baroclinic dynamics, and interactions among large and small flow scales reproduced by high-resolution ocean models. However, due to the small degree of freedom of the model, distribution function for a prediction scale and its high-order moments were computed for a large number of ensemble realizations (up to $10^{5}$ ). That allows us to quantify PE statistics in an accurate manner.

Similar analysis is difficult to realize in full-scale numerical forecast ocean models due to limited computer resources. The full-scale models produce small sample forecast ensembles and therefore, cannot resolve the full complexity of PE statistics. The idealized model reveals some trends in behavior of PE and produces useful knowledge for extracting them from small forecast ensemble samples.

The following trends obtained in the present study need to be examined by baroclinic highresolution ocean models with realistic bottom topography and forcing: 
(1) In the limit of zero horizontal viscosity the model demonstrates the initial decay of spatially uncorrelated and correlated perturbations due to non-linear bottom friction. Since the bottom friction plays a significant role in the energy balance of ocean coastal currents (Wunsch and Ferrari, 2004), the observed decay seems to be an important process that may enhance model predictability.

Initial error decay was noted in connection with numerical experiments in preparation for what became eventually known as the Global Weather Experiment (Williamson and Kasahara, 1971), and more recently, in the oceanographic and meteorological literature, by Brasseur et al. (1996), Wirth and Ghil (2000), Vannitsem and Nicolis (1997), Snyder et al. (2003) among others. The initial error decay found in these models was controlled by dissipative processes parameterized through a hyper-diffusion or usual diffusion operator with a horizontal viscosity. Our study reveals another mechanism of initial error decay: PE decays due to viscosity damping by non-linear bottom friction and this process is weakly dependent on horizontal viscosity.

(2) Statistics of the finite-amplitude prediction error was found to be close to extremum statistics-Weibullian. No general theory is available that demonstrates universality of this result for any forecasting model. However, there is a number of reasons to examine Weibull statistics in large ocean and atmospheric models. First, extremum statistics are often observed to arise in multi-dimensional systems, exhibiting correlation over a broad range of scales, leading to emergent phenomenology, such as self-similarity and in some case fractional dimension (Boffetta et al., 2002). Second, Weibull statistics seems to be a good mathematical tool for the parametrical estimate of PE distributions in small forecast ensembles and from limited observation samples. Preliminary computations provided by us support this conclusion (Ivanov et al., in preparation). Third, if the divergence of a predicted flow in phase space is constant, the cumulative distribution function $P(\bar{\varepsilon}, t)$ (defined by Eq. (5a)) can be analytically estimated (Ivanov et al., in preparation). Here, we only give the final results without calculations:

$$
P(\bar{\varepsilon}, t)=\exp \left(-\frac{c_{0} t}{\langle\tau\rangle}\right),
$$

where $k$ is a constant depending on a forecast model.

CDF (28) corresponds to the exponential probability distribution function, which results from Weibullian distribution (18) for $\gamma=0, \beta=1$, and $\eta=c_{0} /\langle\tau\rangle$. Eq. (28) assesses predictability for many forecast models including Lorenz 63 model (Lorenz, 1963).

(3) Our computations demonstrated that the transition from the linear to non-linear predictability regime may be detected by high-order $\tau$-moment behavior. We found the predictability regime where the mean prediction time scale grew along (quasi)-exponential law, which accompanies the linear predictability, but a behavior of variance for this scale was abnormal, so there are no statistically significant correlations between the forecast skill and ensemble spread. This result explains the lack of correlations between the forecast skill and ensemble spread found in a number of the atmospheric and oceanic models, and may be used to develop criteria for detection of the transition from linear to non-linear predictability regime.

Although the present study has analyzed only model predictability near the equilibrium ocean state, non-monotonous behavior of ensemble spread seems to be a common feature of forecast models. A possible explanation of this effect is that model predictability strongly depends on the 
spatial correlation scale of initial error as it was firstly demonstrated by Lorenz (1965), and on variations of this scale when the PE evolves.

There are in general different scenarios in behavior of the ensemble spread due to variations of the spatial correlation scale of PE. For example, Lorenzo et al. (2003) demonstrated decreasing ensemble spread of an ensemble system consisting of chaotic Lorenz cells diffusively coupled, as the correlation scale of PE grows. If the growing perturbations rapidly adopt the horizontal scales comparable to that of the reference flow, the ensemble spread reaches an asymptotic value and then weakly changes. This case is studied in Section 9.

However independently on the realized scenario, the correlation scale of prediction error should change when the error leaves the tangent space (Schertzer and Lovejoy, 2004). That can, in general, result into variations of high-order statistics of IPT and in the non-monotonous behavior of ensemble spread, examples of which can be found in Nicolis (1992), Cohn (1993) among others. Obviously, a more systematic analytical description of the observed effects is required. We will address this point elsewhere.

\section{Acknowledgements}

The authors are grateful to Prof. Catherine Nicolis at the Royal Meteorological Institute of Belgium for constructive discussion on the stochastic concept of model predictability. Comments from Prof. Lenny Smith at University of Oxford (the United Kingdom) helped to clarify the presentation of the obtained results. The authors would like to thank two anonymous reviewers for helpful comments. This study was supported by the Office of Naval Research and Naval Oceanographic Office for P. Chu, the NATO and NSF (award N OCE-0530748) for L. Ivanov.

\section{Appendix A. Spectral decomposition}

Following Morse and Feshbach (1953), the geostrophic stream function is decomposed into

$$
\Psi=\Psi_{\text {hom }}+\Psi_{\text {harm }}, \quad \Psi_{\text {hom }}(\mathbf{x}, t)=\sum_{m=1}^{M} A_{m}(t) \psi_{m}(\mathbf{x}),
$$

where $\Psi_{\text {harm }}$ is the harmonic function calculated with the open boundary conditions written by

$$
\left.\Psi_{\text {harm }}\right|_{\Lambda^{\prime}}=-\int_{l_{\mathrm{a}}}^{x_{2}} u_{\mathrm{b}}(x, t) \mathrm{d} x \quad \text { and }\left.\quad \Psi_{\mathrm{hom}}\right|_{\Lambda}=0 .
$$

The basis functions $\left\{\Psi_{\mathrm{m}}\right\}$ are the eigenfunctions of the horizontal Laplacian operator $\Delta$ and computed by

$$
\Delta \psi_{\mathrm{m}}=-\lambda_{\mathrm{m}} \psi_{\mathrm{m}},\left.\quad \psi_{m}\right|_{\Lambda \cup \Lambda^{\prime}}=0,
$$

where $\lambda_{\mathrm{m}}$ are its eigenvalues.

Spectral decomposition (A1)-(A3) is also applicable for non-rectangular domains and can be generalized for 2D compressible and 3D incompressible flows (Chu et al., 2003).

The optimal truncation number $M_{\mathrm{opt}}$ in (A1) depends on complexity of a flow structure. Direct computations have shown that to represent the reference flow with accuracy better than $0.1 \%$, the truncation number $M_{\text {opt }}$ should be taken about $10^{2}$.

This number can be theoretically confirmed in the following way. The used hydrodynamic model may resolve ocean flows with $\sim 100-200 \mathrm{~km}$ spatial scales $(L)$ because of numerical grid 
with $50 \mathrm{~km}$ cells. Following Mikhlin (1964) the spatial scale of the highest-order mode $\psi_{\mathrm{m}}$ can be defined as

$$
L_{M} \sim L_{1}\left(\frac{\lambda_{1}}{\lambda_{M}}\right)^{1 / 2}
$$

where $L_{1}$ is the spatial characteristic scale of the largest mode $m=1$.

For the rectangular Domain-A we can suppose $L_{M} \approx L, L_{1} \approx 10^{8} \mathrm{~km}$ and $L \approx 10^{7}$ to $2 \times 10^{7} \mathrm{~km}$. Substituting $L_{1}$ and $L_{\mathrm{M}}$ into Eq. (A4), yields

$$
\frac{\lambda_{1}}{\lambda_{M}} \sim 25-100,
$$

Therefore, from 80 to 120 modes should approximate the reference and perturbed solutions with a reasonable accuracy. We used $M_{\mathrm{opt}}=100$.

\section{Appendix B. Dissipation of prediction error}

Following Blumberg and Mellor (1987), the bottom friction of both velocity components is parameterized by

$$
\begin{aligned}
& F_{1}\left(u_{1}, u_{2}\right)=\alpha E u_{1}, \\
& F_{2}\left(u_{1}, u_{2}\right)=\alpha E u_{2},
\end{aligned}
$$

where $\alpha$ is the drag coefficient, $E=\sqrt{u_{1}^{2}+u_{2}^{2}}$. Let the circulation in a basin be decomposed as the reference circulation $\left(\bar{u}_{1}, \bar{u}_{2}\right)$ and perturbations $\left(u_{1}^{\prime}, u_{2}^{\prime}\right)$ :

$$
u_{1}=\bar{u}_{1}+u_{1}^{\prime}, \quad u_{2}=\bar{u}_{2}+u_{2}^{\prime},
$$

such that

$$
\left|\bar{u}_{1}\right| \gg\left|u_{1}^{\prime}\right| \text { and }\left|\bar{u}_{2}\right| \gg\left|u_{2}^{\prime}\right| \text {. }
$$

For small perturbations, linearization of (B1) and (B2) leads to

$$
\begin{aligned}
& F_{1} \approx F_{1}\left(\bar{u}_{1}, \bar{u}_{2}\right)+\alpha E^{-1 / 2}\left[\left(2 \bar{u}_{1}^{2}+\bar{u}_{2}^{2}\right) u_{1}^{\prime}+\bar{u}_{1} \bar{u}_{2} u_{2}^{\prime}\right], \\
& F_{2} \approx F_{2}\left(\bar{u}_{1}, \bar{u}_{2}\right)+\alpha E^{-1 / 2}\left[\left(\bar{u}_{1}^{2}+2 \bar{u}_{2}^{2}\right) u_{2}^{\prime}+\bar{u}_{1} \bar{u}_{2} u_{1}^{\prime}\right]
\end{aligned}
$$

Therefore, the dissipative terms in the prediction error energy balance can be written by

$$
\begin{aligned}
& \left\langle F_{1} u_{1}^{\prime}\right\rangle \approx \alpha E^{-1 / 2}\left[\gamma_{1}\left\langle\left(u_{1}^{\prime}\right)^{2}\right\rangle+\gamma_{2}\left\langle u_{1}^{\prime} u_{2}^{\prime}\right\rangle\right], \\
& \left\langle F_{2} u_{2}^{\prime}\right\rangle \approx \alpha E^{-1 / 2}\left[\gamma_{3}\left\langle\left(u_{2}^{\prime}\right)^{2}\right\rangle+\gamma_{2}\left\langle u_{1}^{\prime} u_{2}^{\prime}\right\rangle\right],
\end{aligned}
$$

where $\gamma_{1}=2 \bar{u}_{1}^{2}+\bar{u}_{2}^{2}, \gamma_{2}=\bar{u}_{1} \bar{u}_{2}$ and $\gamma_{3}=\bar{u}_{1}^{2}+2 \bar{u}_{2}^{2}$. 


\section{Appendix C. Probability density function of initial perturbations}

Let the two-point correlation function of the geostrophic stream function be projected onto the phase space with the basis $\left[\psi_{1}, \ldots, \psi_{M}\right]$ :

$$
\left\langle\delta \Psi\left(\mathbf{x}_{1}\right) \delta \Psi\left(\mathbf{x}_{2}\right)\right\rangle=\sum_{l=1}^{M} \sum_{m=1}^{M}\left\langle\delta A_{l}^{0} \delta A_{m}^{0}\right\rangle \psi_{l}\left(\mathbf{x}_{1}\right) \psi_{m}\left(\mathbf{x}_{2}\right),
$$

Integrating $(\mathrm{C} 1)$ over the spatial variables $\mathbf{x}_{1}, \mathbf{x}_{2}$ in the computation area yields:

$$
\iint \mathrm{d}^{2} \mathbf{x}_{1} \mathrm{~d}^{2} \mathbf{x}_{2}\left\langle\delta \Psi\left(\mathbf{x}_{1}\right) \delta \Psi\left(\mathbf{x}_{2}\right)\right\rangle=\left\langle\delta A_{l}^{0} B_{l m} \delta A_{m}^{0}\right\rangle,
$$

with

$$
\iint \mathrm{d}^{2} \mathbf{x}_{1} \mathrm{~d}^{2} \mathbf{x}_{2} \psi_{l}\left(\mathbf{x}_{1}\right) \psi_{m}\left(\mathbf{x}_{2}\right)=B_{l m}
$$

Using (10), the correlation function in the left-hand side of (C2) is represented, following Panchev (1971) as

$$
\left\langle\delta \Psi\left(\mathbf{x}_{1}\right) \delta \Psi\left(\mathbf{x}_{2}\right)\right\rangle=\frac{1}{2} R_{\perp}^{2} I^{2} \exp \left(-\frac{\left(\mathbf{x}_{1}-\mathbf{x}_{2}\right)^{2}}{2 R_{\perp}^{2}}\right), \quad R_{\perp} \ll L \sim L_{1} .
$$

Introducing new variables: $\mathbf{r}=\mathbf{x}_{1}-\mathbf{x}_{2}$ and $\mathbf{r}_{1}=\mathbf{x}_{1}+\mathbf{x}_{2}$, and integrating (C2) over $\mathbf{r}$ and $\mathbf{r}_{1}$ yield:

$$
\left\langle\delta A_{l}^{0} B_{l m} \delta A_{m}^{0}\right\rangle=2 \pi L_{1}^{2} L_{2}^{2} R_{\perp}^{4} I^{2} \operatorname{erf}\left(\frac{L_{1}}{\sqrt{2} R_{\perp}}\right) \operatorname{erf}\left(\frac{L_{2}}{\sqrt{2} R_{\perp}}\right) .
$$

Eq. (C5) defines an $M$-dimension hyper ellipsoidal surface $\Omega_{0}$ in the model phase space. The ellipsoid semi-axes are directed along the eigenvectors of the matrix $B_{l m}$. Therefore, the maximum deviation for the vector of initial perturbations $\left\langle\left(\delta A_{\max }^{0}\right)^{2}\right\rangle^{1 / 2}$ is estimated by

$$
\left\langle\left(\delta A_{\max }^{0}\right)^{2}\right\rangle^{1 / 2}=(2 \pi)^{1 / 2} L_{1} L_{2} R_{\perp}^{2} \bar{I} \operatorname{erf}\left(\frac{L_{1}}{\sqrt{2} R_{\perp}}\right)^{1 / 2} \operatorname{erf}\left(\frac{L_{2}}{\sqrt{2} R_{\perp}}\right)^{1 / 2} \lambda_{\max }^{1 / 2},
$$

where $\lambda_{\max }$ is the maximum eigenvalue of the matrix $B_{l m}$.

In the context of the predictability problem, the PDF of a RNLP is Gaussian with the mean $\left\langle\delta A_{m}^{0}\right\rangle=0$ and the covariance matrix:

$$
\sigma_{l m}^{2}=B_{l m} L_{1}^{-2} L_{2}^{-2} R_{\perp}^{-4} I^{-2} \operatorname{erf}\left(\frac{L_{1}}{\sqrt{2} R_{\perp}}\right)^{-1} \operatorname{erf}\left(\frac{L_{2}}{\sqrt{2} R_{\perp}}\right)^{-1} .
$$

\section{Appendix D. Probability weighted moments}

The probability weighted moments of a random variable are defined by Greenwood et al. (1979):

$$
\alpha_{k}=\int_{0}^{1} X(u)(1-u)^{k} \mathrm{~d} u \quad \text { and } \quad \beta_{k}=\int_{0}^{1} X(u) u^{k} \mathrm{~d} u
$$


where $k=0,1, \ldots, K$ and $X(u)$ is the quantile function (i.e., the inverse of cumulative distribution function). Following Hosking and Wallis (1997) $\left(\alpha_{k}, \beta_{k}\right)$ are calculated from an ordered random sample $\tau_{n}$ of size $N$ as unbiased estimates of $\left(\hat{\alpha}_{k}, \hat{\beta}_{k}\right)$ :

$$
\hat{\alpha}_{k}=\frac{1}{N} C\left(\begin{array}{c}
N-1 \\
k
\end{array}\right) \sum_{n=1}^{N} C\left(\begin{array}{c}
N-n \\
k
\end{array}\right) \tau_{n}, \quad \hat{\beta}_{k}=\frac{1}{N} C\left(\begin{array}{c}
N-1 \\
k
\end{array}\right) \sum_{n=1}^{N} C\left(\begin{array}{c}
n-1 \\
k
\end{array}\right) \tau_{n},
$$

where $C\left(\begin{array}{l}p \\ q\end{array}\right)$ are the binomial coefficients.

The following procedure is used to estimate the parameters of a Weibull distribution function.

(a) The probability weighted moments $\alpha_{k}$ or $\beta_{k}$ are computed from modeled samples accordingly to (D2). (b) With the given moments $\left(\alpha_{k}, \beta_{k}\right)$, the Weibull distribution parameters are identified from the condition:

$$
\mathrm{KL}_{\mathrm{s}}(\eta, \beta, \gamma)=\mathrm{KL}\left(F_{1} \mid F_{2}\right)+\mathrm{KL}\left(F_{2} \mid F_{1}\right) \rightarrow \min ,
$$

where $\mathrm{KL}_{\mathrm{s}}$ is the symmetrical Kullback-Leibler distance, $F_{1}$ and $F_{2}$ are distribution functions computed from Monte Carlo samples and identified by the probability weighted moments, respectively.

\section{References}

Aubry, N., Guyonnet, R., Lima, R., 1991. Spatiotemporal analysis of complex signals: theory and applications. J. Stat. Phys. 64 (3/4), 683-739.

Aurell, E., Boffetta, G., Crisanti, A., Paladin, G., Vulpiani, A., 1996. Predictability in systems with many characteristic times: the case of turbulence. Phys. Rev. E 53 (2), 2337-2349.

Benzi, R., Carnevale, G.F., 1989. A possible measure of local predictability. J. Atmos. Sci. 46, 3595-3598.

Berloff, P.S., McWilliams, J.M., 1999. Large-scale, low-frequency variability in wind-driven ocean gyres. J. Phys. Oceanogr. 29 (8), 1925-1949.

Blumberg, A.F., Mellor, G.L., 1987. A description of a three-dimensional coastal ocean circulation model. In: Three Dimensional Coastal Ocean Models. Amer. Geophys. Union, pp. 1-16.

Boffetta, G., Giuliani, P., Paladin, G., Vulpiani, A., 1998. An extension of Lyapunov analysis for the predictability problem. J. Atmos. Sci. 55, 3409-3416.

Boffetta, G., Cencini, M., Falconi, M., Vulpiani, A., 2002. Predictability: a way to characterize complexity. Phys. Rep. $356,367$.

Brasseur, P., Blayo, E., Verron, J., 1996. Predictability experiments in the North Atlantic Ocean: outcome of a quasigeostrophic model with assimilation of TOPEX/POSEIDON altimeter data. J. Geophys. Res. 101 (C9), 14161-14173.

Bury, K., 1999. Statistical Distribution in Engineering. Cambridge University Press, Cambridge.

Chu, P.C., Ivanov, L.M., 2005. Statistical characteristics of irreversible predictability time in regional ocean models. Non. Proc. Geophys. 12, 1-10.

Chu, P.C., Fan, C.W., Ehret, L.L., 1997. Determination of open boundary conditions from interior observational data. J. Atmos. Ocean. Technol. 14, 723-734.

Chu, P.C., Ivanov, L.M., Margolina, T.M., Melnichenko, O.V., 2002. On probabilistic stability of an atmospheric model to various amplitude perturbations. J. Atmos. Sci. 59, 2860-2873.

Chu, P.C., Ivanov, L.M., Margolina, T.M., Korzhova, T.P., Melnichenko, O.V., 2003. Analysis of sparse and noisy ocean current data using flow decomposition. Part 1. Theory. J. Atmos. Ocean. Technol. 20, 478-491.

Cohn, S.E., 1993. Dynamics of short-term univariate forecast error covariances. Mon. Weather Rev. 121, 3123-3149.

Downing, D.J., Gardner, R.H., Hoffman, F.O., 1985. An examination of response-surface methodologies for uncertainty analysis in assessment of models. Technometrics 27 (2), 151-163.

Eremeev, V.N., Ivanov, L.M., Kochergin, S.V., Melnichenko, O.V., 1992. Seasonal variability and types of currents in the upper layer of the Black Sea. Sov. J. Phys. Oceanogr. 3, 193-208.

Freidlin, M.I., Wentzell, A.D., 1998. Random Perturbations of Dynamical Systems. Springer-Verlag. 
Gilmour, I., Smith, L.A., Buizza, R., 2001. Linear regime duration: is $24 \mathrm{~h}$ a long time in synoptic weather forecasting? J. Atmos. Sci. 58 (22), 3525-3539.

Good, P.I., 1996. Re-sampling Methods. A Practical Guide to Data Analysis. Birkhauser, Boston.

Greenwood, J.A., Landwer, J.M., Matalas, N.C., Wallis, J.R., 1979. Probability-weighted moments: definition and relation to parameters of several distributions expressible in inverse form. Water Resour. Res. 15 (5), 1049-1054.

Guckenheimer, J., Holmes, Ph., 1983. Nonlinear Oscillations, Dynamical Systems, and Bifurcations of Vector Fields. Springer.

Holland, W.R., Malanotte-Rizzoli, P., 1989. Along-track assimilation of altimeter data into an ocean circulation model: space versus time resolution studies. J. Phys. Oceanogr. 19, 1507-1534.

Hosking, J.R.M., Wallis, J.K., 1997. Regional Frequency Analysis: An Approach Based on L-moments. Cambridge University Press, Cambridge.

Ivanov, L.M., Chu, P.C., 2006. On stochastic stability of regional ocean models with uncertainty in wind forcing. Non. Proc. Geophys. (revised).

Ivanov, L.M., Chu, P.C., in preparation. Effects of stochastic open boundary on predictability of regional ocean models.

Ivanov, L.M., Kirwan, A.D., Melnichenko, O.V., 1994. Prediction of the stochastic behavior of nonlinear systems by deterministic models as a classical time-passage probabilistic problem. Non. Proc. Geophys. 1, 224-233.

Ivanov, L.M., Collins, C.A., Nicolis, C., in preparation. Statistical estimate in small ensembles and observation samples. Phys. Rev. E.

Kaneko, K., 1998. On the strength of attractors in a high-dimensional system. Milnor attractor: Milnor attractor network, robust global attraction, and noise-induced selection. Physica D 124, 322-344.

Kravtsov, Yu.A., 1993. Limits of Predictability. Springer.

Lacarra, J.F., Talagrand, O., 1988. Short-range evolution of small perturbations in a barotropic model. Tellus A 40, 81-95.

Latin Hypercube, 2007. Matlab Statistics Toolbox 6.0: http://www.mathworks.com/products/statistics/.

Lermusiaux, P.F.J., 2001. Evolving the subspace of the three-dimensional multiscale ocean variability: Massachusetts Bay. J. Mar. Syst. 29 (1-4), 385-422.

Lermusiaux, P.F.J., 2002. On the mapping of multivariate geophysical fields: sensitivity to size, scales and dynamics. J. Atmos. Ocean. Technol. 19, 1602-1637.

Lermusiaux, P.F.J., Robinson, A.R., 1999. Data assimilation via error subspace statistical estimation. Part I. Theory and schemes. Mon. Weather Rev. 127 (7), 1385-1407.

Lermusiaux, P.F.J., Anderson, D.G.M., Lozano, C.J., 2000. On the mapping of multivariate geophysical fields: error and variability subspace estimates. Quart. J. R. Meteorol. Soc. 126, 1387-1430.

Lermusiaux, P.E.J., Chiu, C.-S., Gawarkiewicz, G.G., Abbot, P., Robinson, A.R., Miller, R.N., Haley, P.J., Leslie, W.G., Majumdar, S.J., Pang, A., Lekien, F., 2006. Quantifying uncertainties in ocean predictions. Oceanography 19 (N1), 93-105.

Lorenz, E.N., 1963. Deterministic non-periodic flows. J. Atmos. Sci. 20, 130-141.

Lorenz, E.N., 1965. A study of the predictability of a 28-variable atmospheric model. Tellus 17, 321-333.

Lorenz, E.N., 1984. Irregularity. A fundamental property of the atmosphere. Tellus A 36, 98-110.

Lorenz, E.N., 1996. Predictability-a problem partly solved. In: Proceedings of the Seminar on Predictability. European Center for Medium-Range Weather, Reading, United Kingdom, pp. 1-18.

Lorenz, E.N., 2005. A look at some details of the growth of initial uncertainties. Tellus A 57, 1-11.

Lorenzo, M.N., Santos, M.A., Perez-Munuzuri, V., 2003. Spatio-temporal stochastic forcing effects in an ensemble consisting of arrays of diffusively coupled Lorenz cells. Chaos 13 (3), 913-1049.

Masuda, S., Akitomo, K., Awaji, T., 1999. Effects of stratification and bottom topography on the Kuroshio path variation south of Japan. Part I. Dependence of the path selection on velocity. J. Phys. Oceanogr. 29, 2419-2431.

Mikhlin, S.G., 1964. Variational Methods in Mathematical Physics. Macmillian.

Miller, R.N., Ehret, L.L., 2002. Ensemble generation for models of multimodal systems. Mon. Weather Rev. 130, 2313-2333.

Molteni, F., Palmer, T.N., 1993. Predictability and finite-time instability of the northern winter circulation. Quart. J. R. Meteorol. Soc. 119, 269-298.

Molteni, F., Buizza, R., Palmer, T.N., Petroliagis, T., 1996. The new ECMWF ensemble prediction system: methodology and validation. Quart. J. R. Meteorol. Soc. 122, 73-119.

Moore, A.M., 1999. The dynamics of error growth and predictability in a model of the Gulf Stream. Part II. Ensemble prediction. J. Phys. Oceanogr. 29, 762-778.

Morse, P.M., Feshbach, H., 1953. Methods of Theoretical Physics. McGraw-Hill.

Mu, M., Sun, L., Dijkstra, H.A., 2004. The sensitivity and stability of the ocean's thermohaline circulation to finiteamplitude perturbations. J. Phys. Oceanogr. 34, 2305-2315. 
Nicolis, C., 1992. Probabilistic aspects of error growth in atmospheric dynamics. Quart. J. R. Meteorol. Soc. 118, 553-568. Palmer, T.N., 2000. Predicting uncertainty in forecast of weather and climate. Rep. Progr. Phys. 63, 71-116.

Panchev, S., 1971. Random Functions and Turbulence. Pergamon Press, Oxford.

Pedlosky, J., 1987. Geophysical Fluid Dynamics, 2nd ed. Springer-Verlag, New York.

Robinson, A.R., Haidvogel, D.B., 1980. Dynamical forecast experiments with a barotropic open ocean model. J. Phys. Oceanogr. 10, 1909-1928.

Robinson, A.R., Arango, H.G., Warn-Varnas, A., Leslie, W.G., Miller, A.J., Haley, P.J., Lozano, C.J., 1996. Real-time regional forecasting. In: Modern Approaches to Data Assimilation in Ocean Modeling. Elsevier, pp. 337-409.

Sabel'feld, K., 1991. Monte Carlo Methods in Boundary Value Problems. Springer-Verlag, New York.

Schertzer, D., Lovejoy, S., 2004. Space-time complexity and multifractal predictability. Physica A 338, $173-186$.

Schmeits, M.J., Dijkstra, H.A., 2000. Physics of the 9-month variability in the Gulf Stream region: combining data and dynamical systems analyses. J. Phys. Oceanogr. 30, 1967-1987.

Smith, L.A., Zeiehmann, C., Fraedrich, K.F., 1999. Uncertainty dynamics and predictability in chaotic systems. Quart. J. R. Meteorol. Soc. 125, 2855-2886.

Snyder, C., Hamill, T.M., Trier, S.B., 2003. Linear evolution of error co-variances in a quasi-geostrophic model. Mon. Weather Rev. 131, 189-205.

Stanev, E.V., Staneva, J.V., 2000. The impact of the baroclinic eddies and basin oscillations on the transitions between different quasi-stable states of the Black Sea circulation. J. Mar. Syst. 24, 3-26.

Sura, P., Fraedrich, K., Lunkeit, F., 2001. Regime transitions in a stochastically forced double-gyre model. J. Phys. Oceanogr. 31, 411-426.

Syrovich, L., 1989. Chaotic dynamics of coherent structures. Physica D 37, 126-145.

Toth, Z., 1991. Estimation of atmospheric predictability by circulation analogs. Mon. Weather Rev. 119, 65-73.

Toth, Z., Kalnay, E., 1997. Ensemble forecasting at NCEP and the breeding method. Mon. Weather Rev. 125, 3297-3319.

Vannitsem, S., Nicolis, C., 1997. Lyapunov vectors and error growth patterns in a T21L3 quasi-geostrophic model. J. Atmos. Sci. 54, 347-361.

Veronis, G., 1966. Wind-driven ocean circulation. Part 2. Numerical solutions of the non-linear problem. Deep Sea Res. $13,31-35$.

Vukicevic, T., 1991. Nonlinear and linear evolution of initial forecast errors. Mon. Weather Rev. 119, 1602-1611.

White, H., 1994. Estimation, Inference and Specification Analysis. Cambridge University Press.

Williamson, D., Kasahara, A., 1971. Adaption of meteorological variables forced by updating. J. Atmos. Sci. 28, 1313-1324.

Wirth, A., Ghil, M., 2000. Error evolution in the dynamics of an ocean general circulation model. Dyn. Atmos. Ocean 32 , 419-431.

Wunsch, C., Ferrari, R., 2004. Vertical mixing, energy, and the general circulation of the oceans. Ann. Rev. Fluid Mech. 36, 281-314. 